\title{
Boundary Extension: The Role of Magnification, Object Size, Context, and Binocular Information
}

\author{
Marco Bertamini and Luke A. Jones \\ University of Liverpool
}

\author{
Alice Spooner \\ University of Central Lancashire
}

\author{
Heiko Hecht \\ Johannes Gutenberg-Universität Mainz
}

\begin{abstract}
Boundary extension is a tendency to remember close-up scenes as if they extended beyond the occluding boundaries. The authors explored the contributing factors using brief retention intervals and computergenerated images. Boundary extension turns out to be more complex than previously thought and is not linked to the effects of image magnification and field-of-view changes. Although this is consistent with the idea that boundary extension is the product of the activation of a mental schema that provides information of what is likely to exist outside the picture boundaries, the authors also found that properties of the object at the center of the picture can affect boundary extension independently of the information at the boundaries. In a test of boundary extension using stereograms, the effect does not seem to depend on amount of perceived depth, suggesting a weaker link to perception of space than previously hypothesized.
\end{abstract}

Keywords: naive physics, boundary extension, pictorial perception, space

The experiential world around humans is continuous; it has depth and is populated by solid objects. Scientists have been interested for centuries in how humans construct this world of solid shapes on the basis of the retinal image. Understanding how people perceive pictures may hold a key to solve the riddle. The fact that flat pictures like photographs and line drawings convey a sense of space points to the nature of the reconstruction. Traditionally, the experienced depth has been attributed to pictorial cues such as perspective and relative size. However, the space perceived within a photograph may not be fundamentally different from the space seen through a window. We use the phenomenon of boundary extension to support this notion.

In the literature, boundary extension (BE) has been researched as a phenomenon in its own right. However, BE and its ramifications for perception can only be understood if we investigate to what extent this memory phenomenon is germane to picture perception and to what extent it interacts with depth perception, that is, the reconstruction of the 3-D world on the basis of the retinal image. Intraub and Richardson (1989) introduced the name bound-

Marco Bertamini and Luke A. Jones, Department of Psychology, University of Liverpool, Liverpool, United Kingdom; Alice Spooner, Department of Psychology, University of Central Lancashire, Preston, United Kingdom; Heiko Hecht, Psychologisches Institut, Johannes GutenbergUniversität Mainz, Mainz, Germany.

This work was supported by Economic and Social Research Council Grant RES-000-22-0169. We thank Jenna Mallows for help in preparing the stimuli and running some of the experiments.

Correspondence concerning this article should be addressed to Marco Bertamini, Department of Psychology, University of Liverpool, Eleanor Rathbone Building, Bedford Street South, Liverpool L69 7ZA, United Kingdom. E-mail: m.bertamini@liv.ac.uk ary extension for a phenomenon observed when participants try to remember a visual scene. There is a tendency to remember more information of the scene than was made visible by the boundaries, especially when the scene was originally presented as a close-up image. According to Intraub (2004), BE reflects an anticipatory projection by the observers, meaning that they remember a portion of the expected space as having been seen. This type of expectation applies to the boundaries of a photograph as much as to regions of a scene outside foveal view, for which a much poorer spatial sampling is available. In this sense, space perceived within a photograph is not different from the space seen in all other conditions by a human observer. This is an important claim, and unfortunately it can only be tested indirectly given that the nature of the phenomenon (BE) requires the use of some type of occlusion boundaries. In this article, we attempt to determine whether $\mathrm{BE}$ is a general perceptual phenomenon, whether it is a measure of perceived space in an image, or whether it is inherently pictorial. We always present pictures with clear boundaries, but we manipulate a number of factors that are linked with perception of space, like binocular disparity, and some that are not, like size of objects.

\section{What Is Boundary Extension?}

Intraub and Richardson (1989) presented to students a set of photographs for $15 \mathrm{~s}$ each. Afterward the students were asked to draw what they remembered of the photographs as accurately as possible. A large majority of the participants drew the objects smaller and included additional information that was not visible inside the borders of the original photograph. This increase in what was remembered in the photograph is believed to be due to an extension of what is represented, and this extension goes beyond the boundaries of the photograph. 
Subsequent studies have documented that BE is a robust effect. $\mathrm{BE}$ can be revealed by recognition as well as reproduction (Intraub, Gottesman, \& Bills, 1998; Intraub \& Richardson, 1989), does not require the presence of partly cropped objects (Intraub, Bender, \& Mangels, 1992), and exists for outline drawings as well as photographs as long as there is a background present or imagined (Intraub et al., 1998; Legault \& Standing, 1992). BE does not require long retention intervals (Intraub et al., 1992); indeed BE is found when the test images are presented after a retention interval of only $1 \mathrm{~s}$ (Intraub, Gottesman, Willey, \& Zuk, 1996). It is robust for both neutral and emotional photographs (Candel, Merckelbach, \& Zandbergen, 2003), and it exists for observers of various ages (Seamon, Schlegel, Hiester, Landau, \& Blumenthal, 2002). BE is not present for object boundaries as opposed to view boundaries, for example, for a hat on top of a shirt (Gottesman \& Intraub, 2003). Finally, BE is greatest for proportional changes in both objects size and spacing (Prenz \& Gerbino, 2004).

$\mathrm{BE}$ was first introduced as a memory effect. However, after contrasting different possibilities, Intraub et al. (1992) argued in favor of a perceptual schema hypothesis. The following four factors need consideration (but are not exclusive):

1. Memory schema. It is possible that in memory the representation moves toward a prototypical view. This effect should increase with the length of the retention interval. There may be two types of prototypes: a prototypical object size or a prototypical viewing distance.

2. Perceptual schema. Unlike with memory schema, the perceptual effect happens immediately, as the scene is viewed. BE therefore should not increase with length of retention interval. In addition, the perceptual schema does not predict any boundary reduction. Intraub et al. (1992) argued in favor of the perceptual schema hypothesis. BE is not due to a memory normalization process but is instead the result of the activation of a perceptual schema that provides information of what is likely to exist outside the picture boundaries. This idea is related to Hochberg's (1986) work about how information from successive glimpses is integrated. Gottesman and Intraub (2002) have found further evidence to support the perceptual schema hypothesis.

3. Object completion. If the boundaries are occluders, viewing a bounded scene is very similar to viewing occluded shapes (e.g., Kanizsa, 1979). In the case of occluded shapes, people perceived the object as completed behind the occluder (amodal completion). This link is appealing, but occluded objects at the boundary are not necessary for BE. Still, one might argue that the background surfaces are occluded. Moreover, nonoccluding object boundaries (i.e., boundaries that do not belong to an aperture) do not lead to BE (Gottesman \& Intraub, 2003).

4. Magnification and minification. Perhaps when a scene is magnified and depth is compressed, a normalization process might account for BE. Critically, BE should be present only for magnified images, but not for close-up views when no magnification has been applied.

\section{Is $\mathrm{BE}$ a Picture Perception or a Space Perception Phenomenon?}

If $\mathrm{BE}$ is indeed the witness of a general space perception phenomenon rather than an idiosyncrasy of picture perception, then the following hypotheses should be true. First, the magnitude of $\mathrm{BE}$ should be independent of magnification of the scene, which is merely a pictorial manipulation. Second, BE magnitude should also be unaffected by the presence of more or less clutter. Likewise, BE should not depend on object size. There is no reason why scenes populated by large objects (like a horse) or small objects (like a mouse) should differ if the layout of the scene is otherwise identical. Third, if BE is a general perceptual phenomenon, then the effect should be even stronger for monocular viewing as this is believed to increase the perception of depth in pictures. Conversely, if $\mathrm{BE}$ is a picture perception phenomenon it should be weaker for monocular viewing because this manipulation makes pictures less like pictures and more like any other scene. Finally, if the aperture is a window and there is consistent binocular stereo information and therefore an increased sense of space, BE should increase.

A related question is why BE should be specific to (or larger for) close-up views of a scene. Intraub et al. (1992) suggested that with close-up images, much of the expected surrounding area is not contained in the picture, so this expected information plays a larger role for close-up images. But how do we define a close-up view? We know that BE is present for pictures that independent observers classify as close-up, but are there objective features in the image that distinguish close-up from far views? This question relates to the distinction between a prototypical view, perhaps one in which the central target occupies a fixed proportion of the image, and a prototypical distance, perhaps one compatible with the action space of the observer.

Our experiments shed light on all of these questions. (a) Magnification: Experiments $1 \mathrm{a}$ and $1 \mathrm{~b}$ test whether magnification and therefore depth compression lead to $\mathrm{BE}$ or to a significantly increased BE. (b) Clutter and object size: Experiment 2 tests the effect of clutter, which is also interesting because close-up images tend to show fewer items. Experiments 3 and 4 test the importance of target size. In Experiment 5, we measured BE when withinsubject memory normalization toward an average viewing distance was impossible. This is important because memory normalization effects probably are copresent with BE effects in most experiments. (c) Monocular viewing: Experiment 6 tested BE under binocular and monocular viewing conditions. (d) Stereo: Finally, in Experiment 7, the same scenes were presented as stereograms.

To anticipate the results, we found an interesting but complex pattern. We confirmed that BE exists for close-up views but that it is unrelated to the extent of the field of view of the picture, therefore BE is not an artifact linked to magnification. We found some memory normalization effects under some conditions (high uncertainty), but these in themselves cannot explain the existence of BE. We found that the central object in the image plays an important role, even when the context is kept constant. If BE were directly related to perceived space we would expect larger BE for monocular viewing and for stereograms. Instead, BE was found to be larger when one is free viewing photographs, compared with when one is inspecting the same images monocularly. BE was present but not significantly different for pictures and for stereo- 
grams. We draw two general conclusions. The first is that BE is not confined to picture perception, rather it is a more general perceptual phenomenon related to scene perception. The second finding is that the first conclusion needs to be qualified: Conditions for $\mathrm{BE}$ need to be right, such as the perceived distance of the objects in the scene (close-up) and the size of the objects. Even though BE is related to perceived space, it does not depend directly on factors, such as binocular information, that contribute to depth perception.

\section{General Method}

We created seven 3-D models of rooms with furniture using the graphic package MAXON Cinema 4D (MAXON, GmbH, Friedrichsdorf, Germany). Figure 1 shows the seven rooms used in Experiment 1: two bedrooms, two offices, a kitchen, a dining room, and a living room. All 3-D objects were drawn in house or are available royalty free on the Internet. We placed a target object near the far wall. We selected seven different objects for the seven rooms, and they were roughly as high as they were tall. The images in Figure 1 were taken from a virtual vantage point at 500 $\mathrm{cm}$ from the target object and $160 \mathrm{~cm}$ from the floor. ${ }^{1}$

All experiments were run on a Macintosh computer in a quiet room. The program that displayed the images was developed in $\mathrm{C}$ language by us. The monitor was a Mitsubishi Diamond90e with a resolution of 1,280 pixels $\times$ 1,024 pixels at $75 \mathrm{~Hz}$.

In all of the experiments, participants were required to inspect three images on a computer screen of three different rooms (e.g., a bedroom, a dining room, and a kitchen), and subsequently they were presented with one of the three (e.g., the bedroom). Participants were asked to judge whether the last image of the bedroom was identical to the one that they saw before or whether the last image was showing the same scene from closer or farther distance than the original. As in Intraub et al. (1996), the study phase included three scenes to force an encoding of the scene as a whole and to avoid the possibility of a simple shape matching. The actual position of the images on the screen had a random shift (translation) of approximately 30 arcmin of visual angle to discourage people from focusing on the location on the monitor and also to make the change from one image to the next less meaningful. In other words, the translation made it extremely unlikely that people could see the room as remaining the same room with a change of furniture and of color scheme.

The experimenter explained the task. There were 12 practice trials after which the participant was asked whether the instructions were clear before the experiment started.

Participants inspected the three scenes by pressing the space bar to go from one to the next. Thus, the study phase was self-paced. There was a retention interval of $1 \mathrm{~s}$ during which a black-and-white checkerboard image was presented as a mask. After the mask, the test image was presented, and participants rated this image by clicking on a response scale at the bottom of the screen. There were five options: The test image could be judged much closer than standard, a bit closer than standard, the same, a bit farther than standard, and much farther than standard. For the purpose of analysis, these five options were coded as $-2,-1,0,1$, and 2, respectively. Negative values were therefore an indication that, on average, the test image was judged closer than the standard, as is typically the case when BE is present. Positive values would suggest that the test image was judged to be farther than the standard, something that can be referred to as boundary contraction. Between trials, a fixation mark was presented for $1 \mathrm{~s}$. The procedure is described in Figure 2.

We modeled this methodology on Intraub et al. (1996) but with some important modifications. One difference was that our stimuli were always virtual photographs, meaning that they were snapshot of 3-D models of familiar environments. We used seven different rooms with different furniture and different light sources but of identical size (see Figure 1). One advantage of using 3-D models is that it is easy to manipulate the two variables of interest: the location of the vantage point and the magnification factor. The studies on BE in the literature tend to use large numbers of participants and few repetitions. Our design required participants to make a larger set of responses to a limited number of scenes (because we have only seven different rooms). The hypothesis was that if $\mathrm{BE}$ is a robust perceptual effect, the fact that a room may become familiar during the experiment should not prevent $\mathrm{BE}$.

\section{Experiment 1a: Is Magnification a Cause of BE?}

As explained in the General Method section, we placed a target object on a pedestal at one end of each room so that distances could be measured with respect to this target. The main hypothesis tested by this experiment was about the role of magnification. We used three levels of magnification, corresponding to $42.74^{\circ}$, $30.01^{\circ}$, and $16.14^{\circ}$ of visual angle horizontally.

To manipulate the view, the existing studies in the literature have relied mainly on the use of different camera lenses. For instance, Intraub et al. (1992) used a zoom lens, and only when the lens was insufficient on its own did they move the tripod forward. If the close-up stimuli are obtained by magnification, which is the result of using a telephoto lens, then it is possible that the magnification is an important factor. If $\mathrm{BE}$ is dependent on a magnification effect, we expected to find a significant BE effect only when the target image was magnified but not when the target was viewed at shorter distances. On the other hand, if BE is not dependent on magnification and if our virtual rooms are reasonably convincing, we expected $\mathrm{BE}$ to be present only for close-up images of both kinds (high magnification and short distance from the target object). Therefore, we predicted $\mathrm{BE}$ to increase as the distance from the target decreases (from $500 \mathrm{~cm}$ up to $200 \mathrm{~cm}$ ) but also as magnification increases.

\section{Method}

Design and materials. In each room there was a rectangular pedestal $120 \mathrm{~cm}$ in height and a different target object in each room (see Figure 1). There were two separate conditions (distance and magnification) with two groups of participants. By distance, we mean the location of the virtual vantage point and a field of view determined by the use of a normal lens. In the distance condition, there were three vantage point distances (500, 350 , and $200 \mathrm{~cm}$ from the target object). The levels of magnification were chosen to match as much as possible the change in size of the target object in the distance stimuli. We started with the $500-\mathrm{cm}$ stimuli and magnified the image by $46 \%$ and $176 \%$. Figure 3 shows a comparison of the distance and the magnification stimuli for one of the rooms. Because changes were matched, and to compare the levels across the two manipulations, we also used the generic term proximity (near, intermediate, and far) to refer to the three levels in terms of the retinal size of the target object (see Figure 3).

Each trial included the presentation of the images of three different rooms in the center of a gray screen. All three images shared the same level of distance or magnification (e.g., $500 \mathrm{~cm}$ distance), as shown in Figure 2.

\footnotetext{
${ }^{1}$ Because these are 3-D models, the measurements are only meaningful as absolute values if the objects are familiar. That is, a snapshot cannot include scale information; however, the same is true of real photographs. A photograph of a small room cannot be discriminated from a photograph of a huge room in which everything has been scaled by the same factor. We assumed that the 3-D models were perceived as normal rooms. We located the height of the vantage point at the standing eye height of an observer 180 $\mathrm{cm}$ tall. This is close to what has been found to be a default value for eye height in picture perception (Hagen \& Giorgi, 1993; Rogers, 2003).
} 

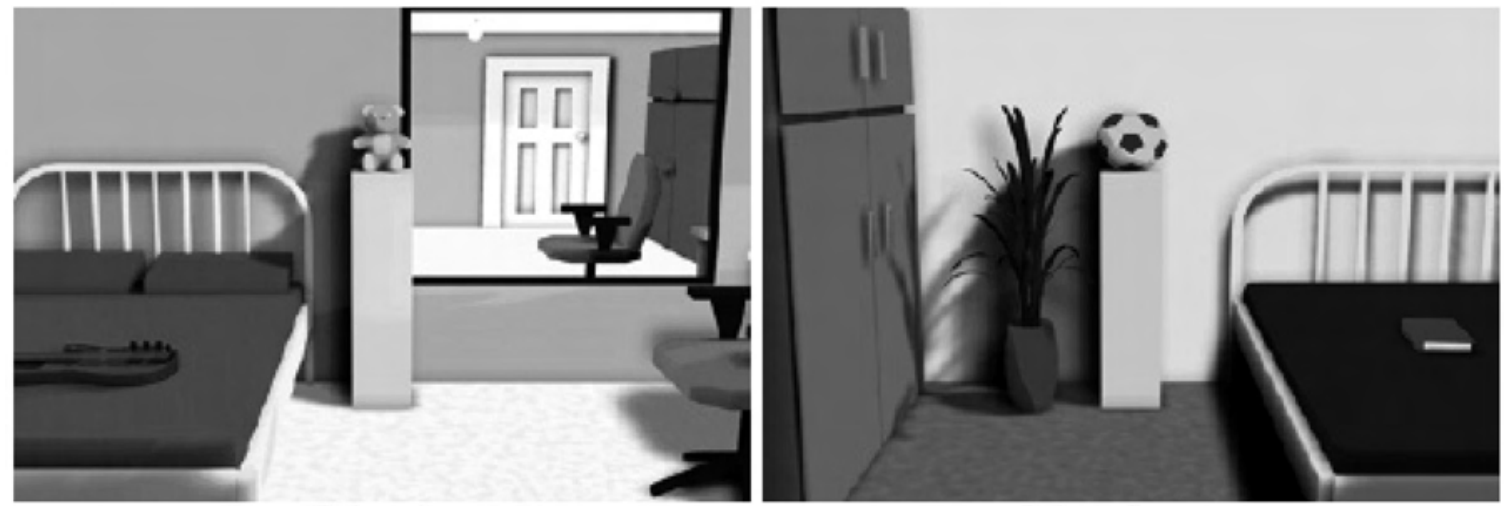

Bedroom 1

Bedroom 2
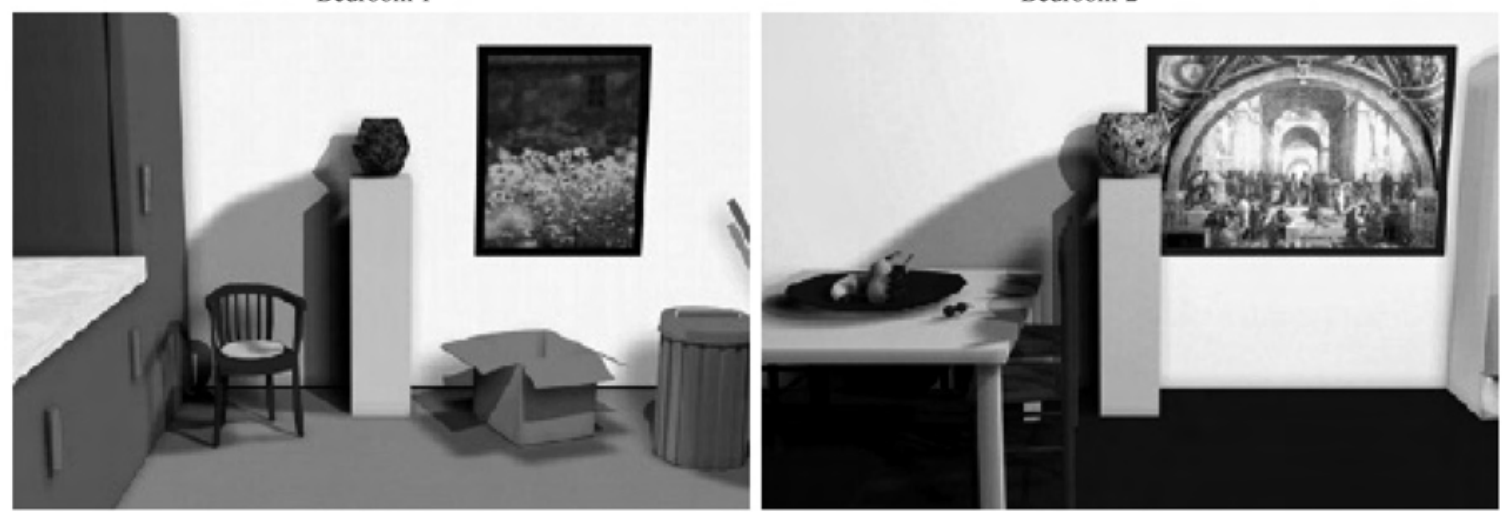

Kitchen

Dining room

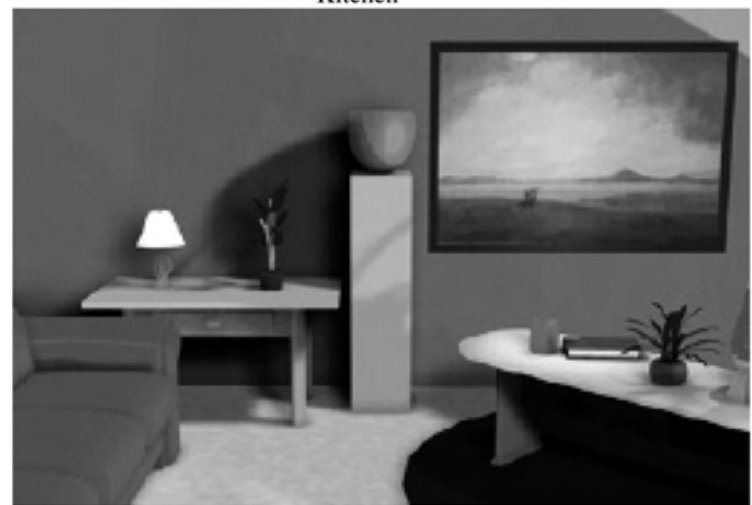

Living room

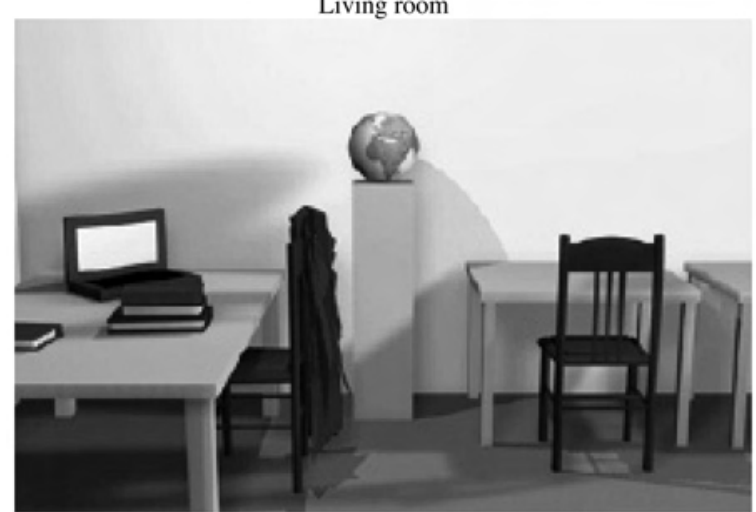

Office 2

Figure 1. Snapshots of the seven rooms used in all experiments. In particular, these images show the rooms as they appeared in Experiment 1 . The vantage point is at $500 \mathrm{~cm}$ from the target object, and the field of view corresponds to that taken with a camera and a normal lens (focal length $=46 \mathrm{~mm}$ ). 


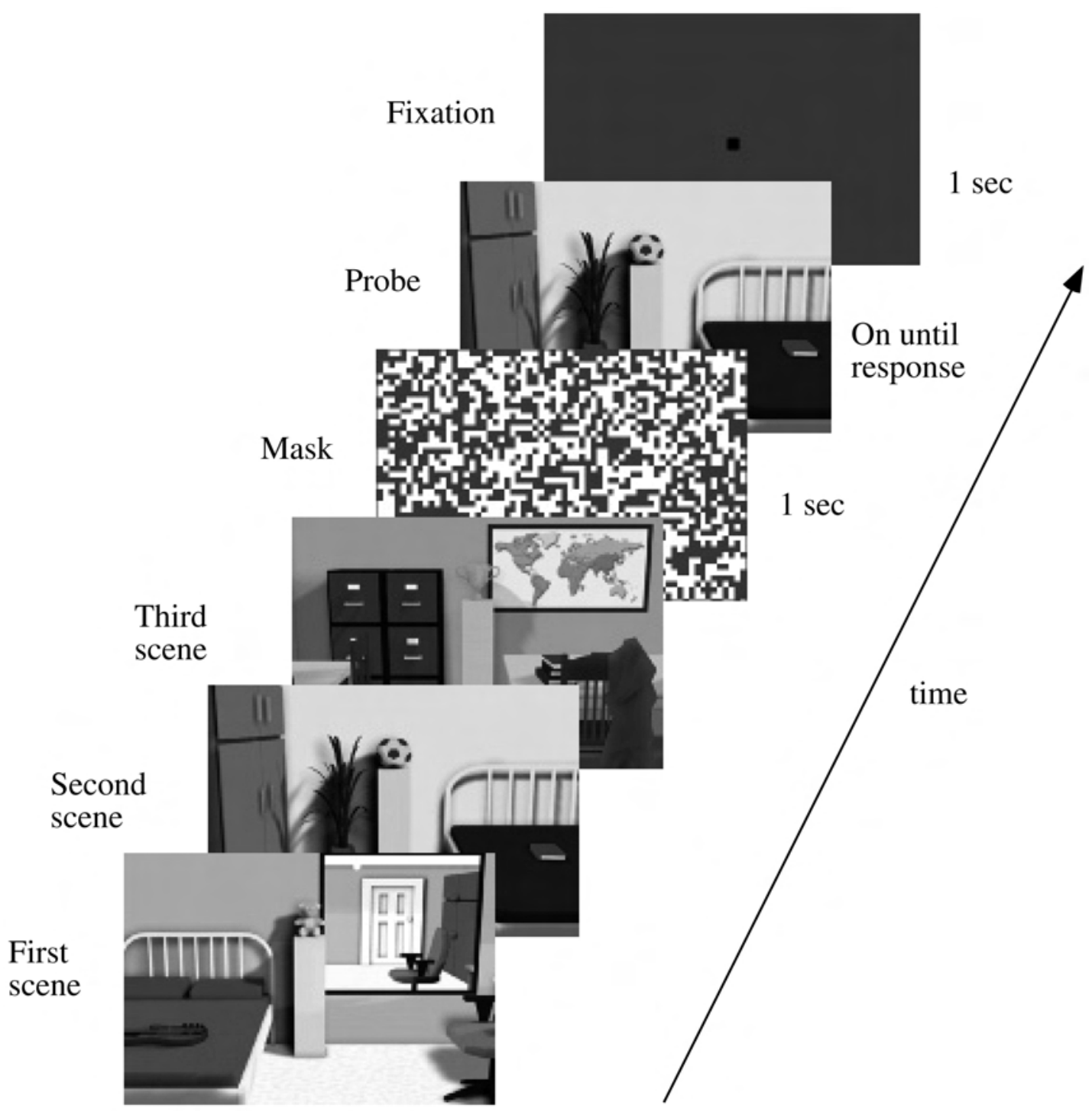

Figure 2. The procedure used in all experiments. The observer studied three images from three different rooms; one of the three room was then presented again after $1 \mathrm{~s}$. The participant would respond by clicking with the mouse on a response scale at the bottom of the screen.

The seven rooms and three levels of either distance or magnification gave 21 test images. Each of these images was also modified to create a test image that could show a bit more or a bit less of the visible scene. For the distance condition, the small changes were shifts of the vantage point by either $15 \mathrm{~cm}$ toward or away from the target object. For the magnification condition, the changes were obtained by scaling up and down by $3 \%$ the size of the image. This value was chosen because it meant that the change in the size of the target object was close to the $15-\mathrm{cm}$ shift of the vantage point. Therefore, although different in nature, we expected these changes to lead to tasks of similar difficulty.

The combination of seven rooms, three distances (or magnifications), and three changes for the test image gave 63 different trials. Each trial was repeated three times in a session for a total of 189 trials per participant.

Participants and procedure. Forty-four participants from the University of Liverpool (Liverpool, United Kingdom) community took part (22 in the distance condition and 22 in the magnification condition).

\section{Results and Discussion}

Figure 4 shows mean responses for different types of test images. Because of how responses were coded, a simple measure of $\mathrm{BE}$ is the overall mean response. A negative mean response indicates that the image was judged to be too close compared with the original (Intraub et al., 1996). The values of this variable can vary between -2 (the participants always judged the image to be much closer) to +2 (the participants always judged the image to be much farther), and a value of 0 indicates no bias. Figure 5 shows the mean response plotted against the change from study image to test image, and a summary of the slopes. The slope depends on how observers used the scale, for instance, whether they did use the two extremes provided, which may depend on their degree of con- 
Distance

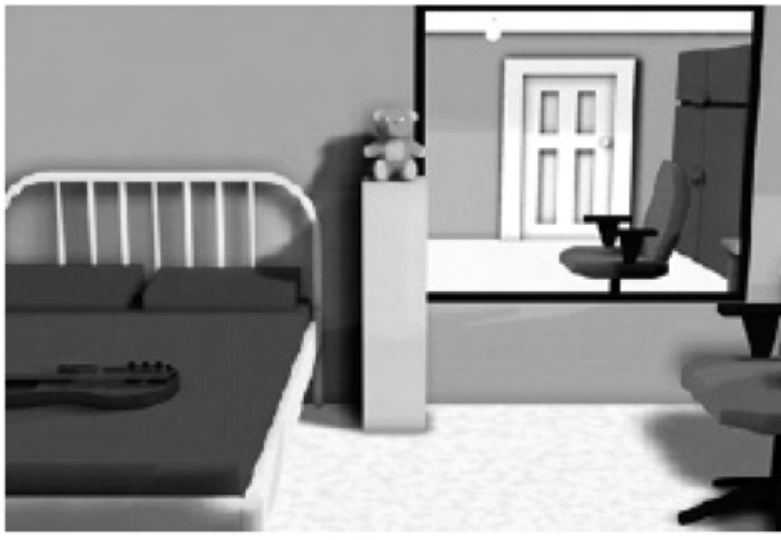

$500 \mathrm{~cm}$

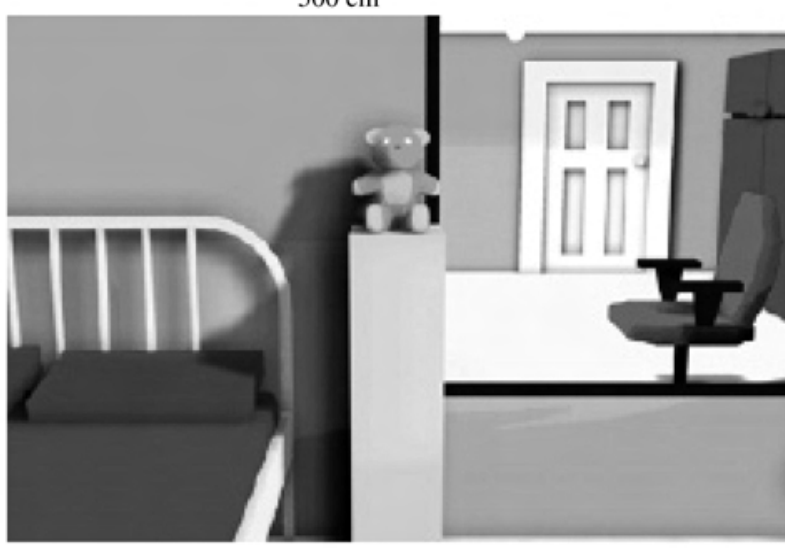

$350 \mathrm{~cm}$

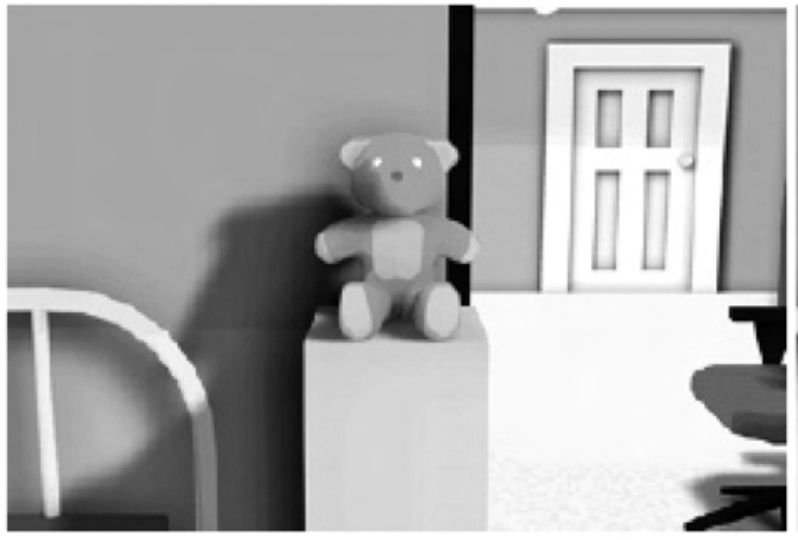

$200 \mathrm{~cm}$
Magnification

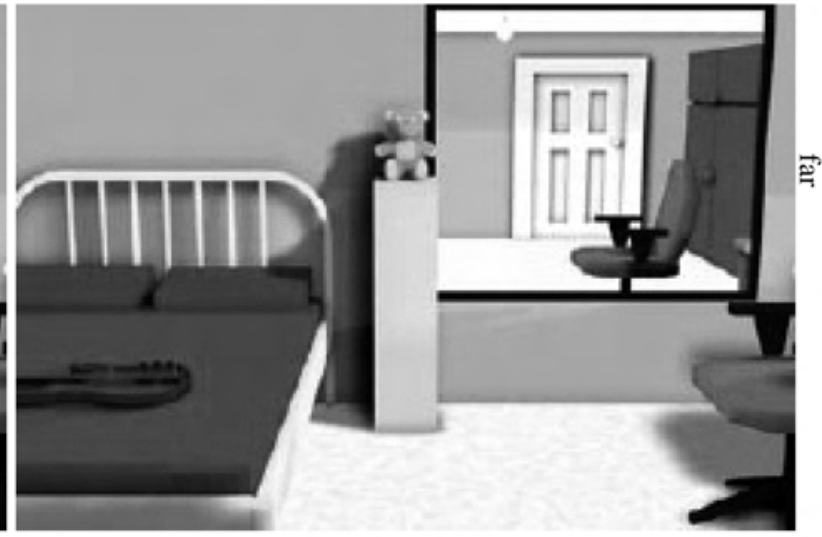

No magnification

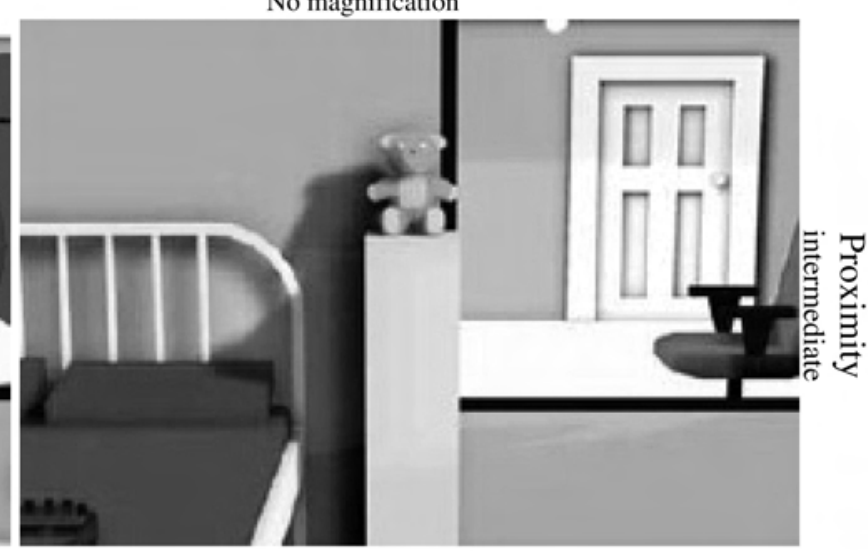

Magnification $46 \%$

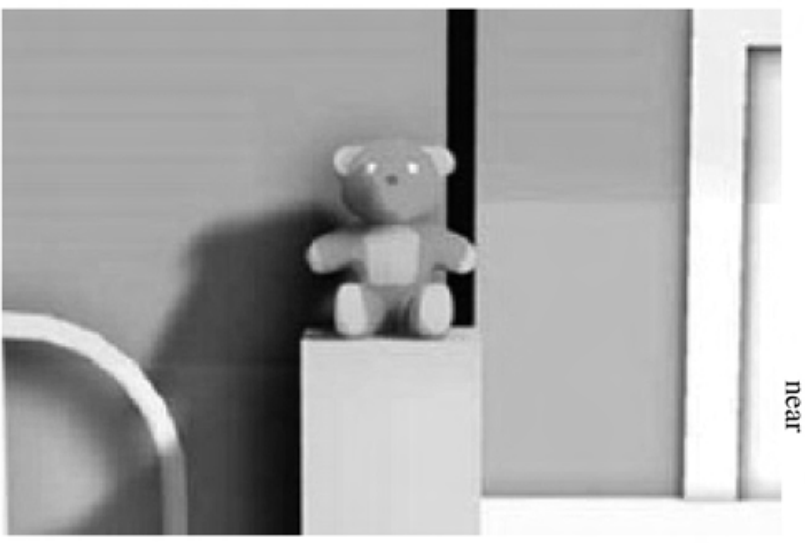

Magnification $176 \%$

Figure 3. Comparison of the distance (left) and magnification (right) manipulations used in Experiment 1. Of the seven rooms, Bedroom 1 is used as an example. Note that although the $200-\mathrm{cm}$ image differs substantially from the image with a $176 \%$ magnification, the size of the teddy bear is matched. The labels under what we call proximity are used as a generic term to refer to either distance or magnification changes.

fidence in their answer. The slope can also be seen as a measure of sensitivity. The maximum theoretical value is 4 (from -2 to +2 ), and a value close to zero would indicate that observers could not perform the task. Perhaps not surprising, the hardest condition seems the one with the greatest magnification (176\%). This may be because little of the room remains visible around the object. It is important to compare Figures 5 and 4, to see whether BE is always associated with low shallow slopes. 

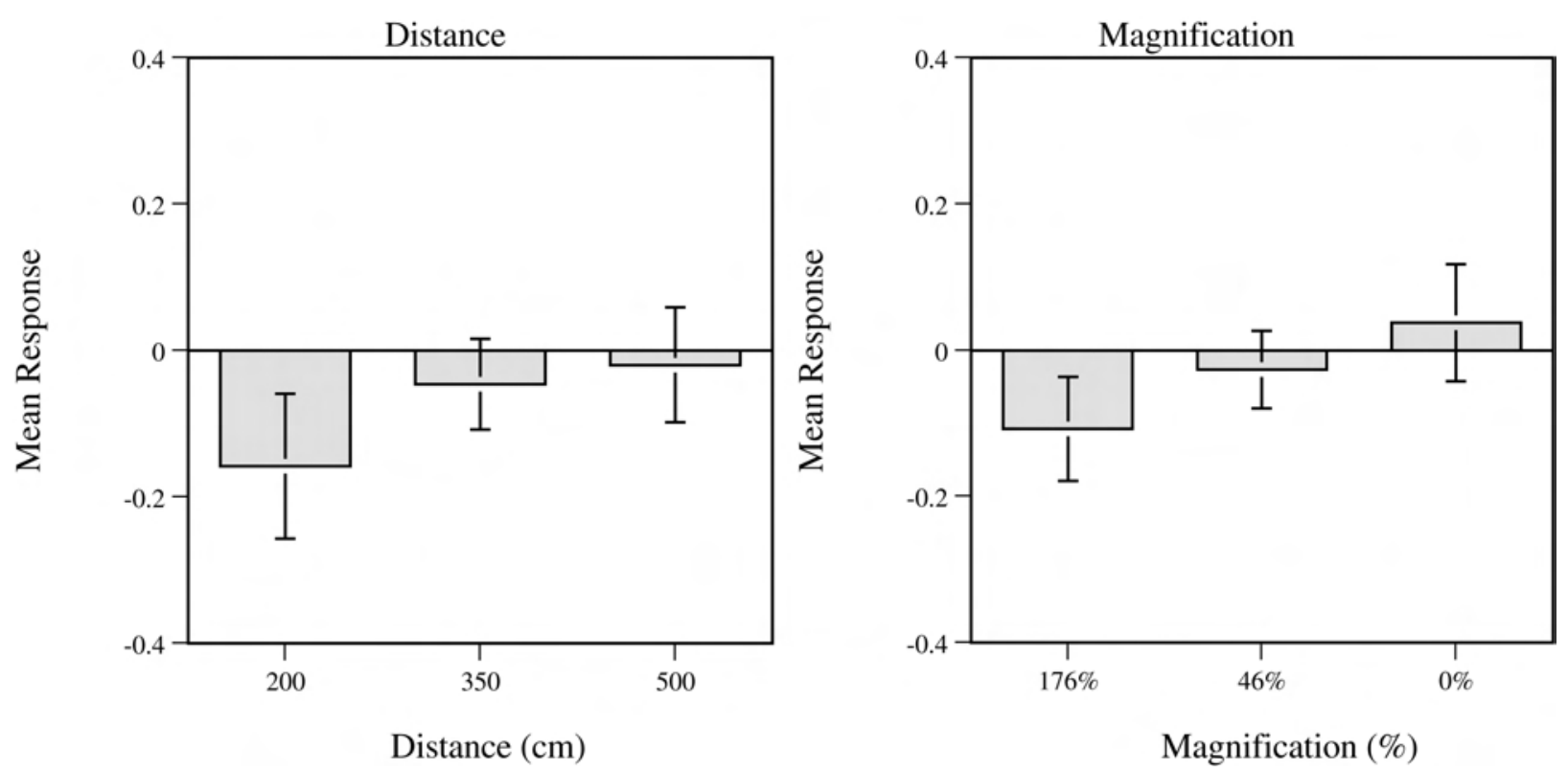

Figure 4. Data for Experiment 1a. Overall mean responses for the three levels of distance and magnification. A negative value is a measure of boundary extension, and a positive value is a measure of boundary contraction. Error bars are $95 \%$ confidence intervals.

There does not seem to be such association; in fact, the $200-\mathrm{cm}$ condition has both the largest negative mean and the steepest slope.

We ran a mixed analysis of variance (ANOVA) on mean response, with manipulation (distance vs. magnification) and proximity (near, intermediate, and far) as independent variables. The analysis confirmed an effect of proximity, $F(2,84)=8.18, p<$ .01 ; no effect of manipulation, $F(1,42)=2.00$; and no interaction between the two, $F(2,84)=0.15$. In the experiments we used seven different rooms. Therefore, we ran an item analysis with respect to the rooms and with the same independent variables as in the first ANOVA. The analysis confirmed an effect of proximity, $F(2,24)=15.66, p<.001$; no effect of manipulation, $F(1,12)=$ 1.79; and no interaction between the two, $F(2,24)=0.31$.

The mean response was -0.13 for the near condition, 0.04 for the intermediate condition, and 0.01 for the far condition. We wanted to test these values against a mean of zero to make sure that a significant BE effect was present. One-sample $t$ tests confirmed that the mean response for the near condition, $t(43)=4.55, p<$ .001 , was significantly different from zero in the negative direction; this is consistent with BE. The far and intermediate conditions were not significantly different from zero.

It is interesting to compare our stimuli with viewing a scene under natural condition, for example, in a window, or viewing a photograph. Our images were computer-generated rooms and could not be mistaken for real photographs. This in itself was not an obstacle to finding a BE effect, presumably because the scene was recognized as a room. More important, the size of the image on the screen ( $9 \mathrm{~cm}$ wide) was similar to that of a photographic print, therefore the viewing conditions were similar to inspecting a photograph. As for most photographs the vantage point of the observer was farther than the correct viewing distance. When we varied the virtual distance from the target object (distance condition), the correct viewing distance of the photograph was always $115 \mathrm{~mm}$. However, in the magnification condition the correct distances were $115 \mathrm{~mm}, 165$ $\mathrm{mm}$, and $317 \mathrm{~mm}$, respectively, for the three levels of magnification. This is another way of describing the difference between moving the camera closer to an object or using a different lens. Conversely, the higher level of magnification under our conditions was less extreme in terms of a mismatch between correct and actual viewing distances. Nevertheless, our conditions were not at all unlike natural viewing of photographs, and the question whether $\mathrm{BE}$ will be present for both types of close-up still remains an important one.

In summary, looking at Figures 4 and 5 again, it is clear that participants could perform the task with some accuracy (as indicated by the slopes) and that there was a shift toward "close" responses (as indicated by the negative means). The analyses confirmed that there was a small but significant BE effect for the $200-\mathrm{cm}$ condition as well as for the $176 \%$ magnification condition. There was no difference between the distance and the magnification conditions. We conclude that BE is not an artifact of the image magnification. In addition, we found no evidence of any boundary contraction.

\section{Experiment 1b: Within-Subjects Manipulation of Magnification and Distance}

This experiment used the same methodology as in Experiment $1 \mathrm{a}$ and can be seen as a control. We wanted to know whether it was possible to obtain a BE effect when the distance and magnification stimuli were presented interleaved and to the same group of participants. 


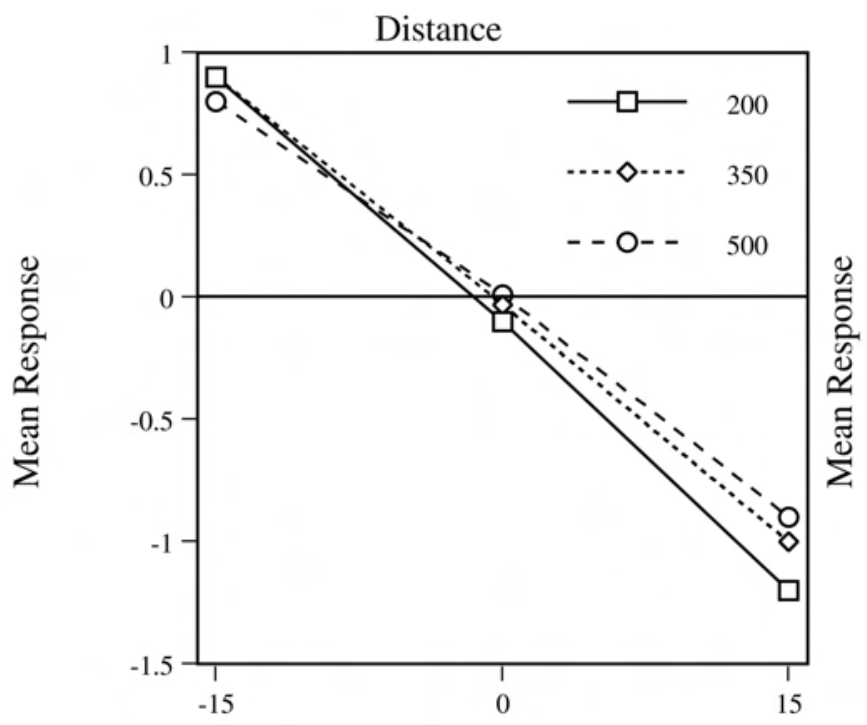

Change from study to test $(\mathrm{cm})$

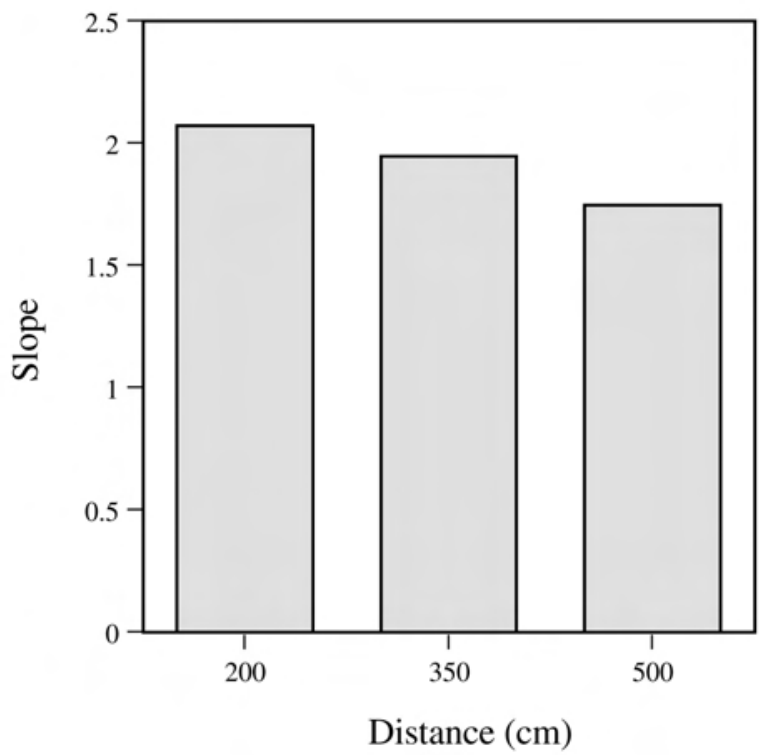

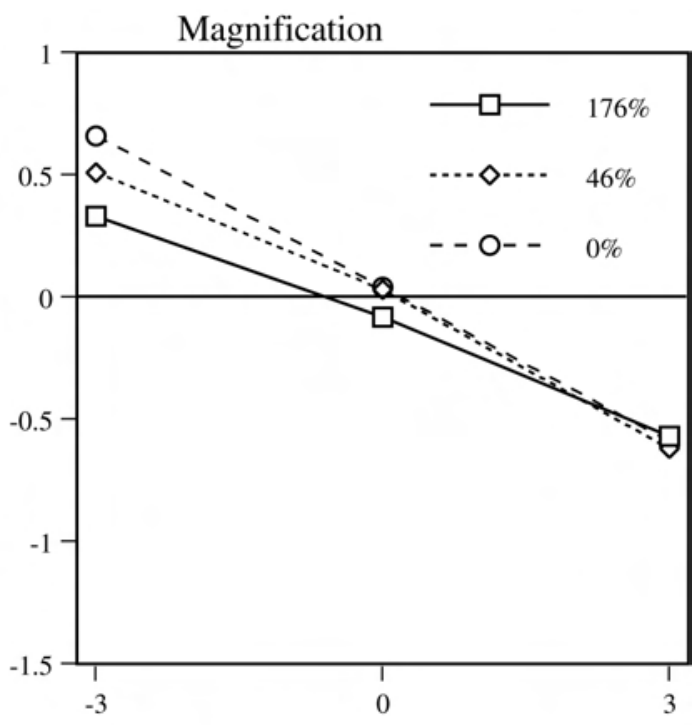

Change from study to test (\%)

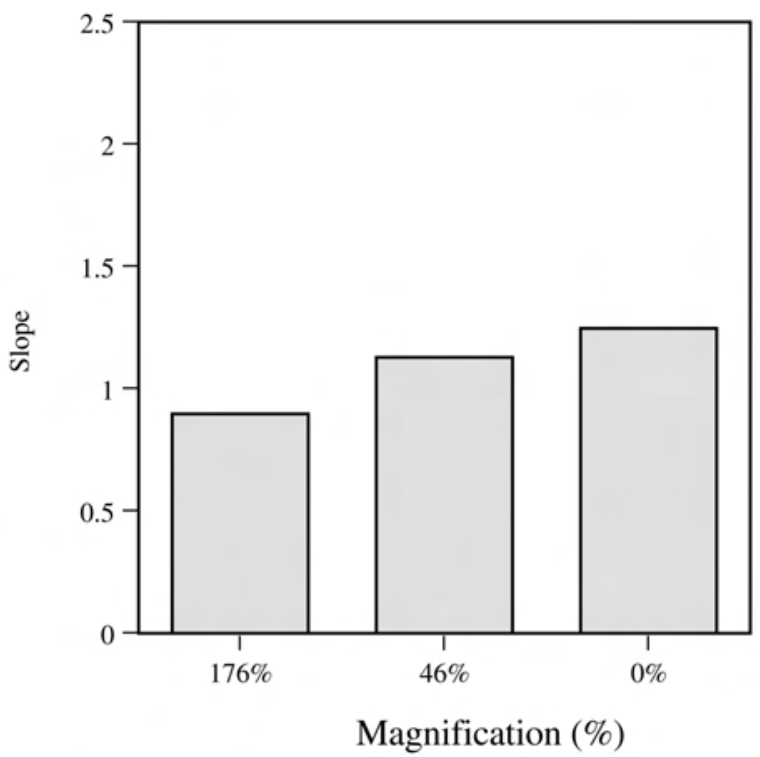

Figure 5. Data for Experiment 1a. Top: Mean responses are plotted for different changes of the test image and separately for the three levels of distance and magnification. Bottom: The performance shown in the line graph above is summarized in terms of slopes. The slope can be taken as an indication of how well observers could perform the task (sensitivity). Because our dependent variable combines accuracy with confidence, high accuracy can lead to relatively shallow slopes if an observer does not use the extremes of the scale; however, using the extremes without accuracy would not lead to steep slopes.

\section{Method}

Design and materials. The stimuli were a subset of the stimuli used in Experiment 1a. We used the $500-\mathrm{cm}$ and $200-\mathrm{cm}$ stimuli from the distance condition and mixed them with the $176 \%$ stimuli from the magnification condition. Therefore, all participants saw both kinds of close-up stimuli but did not see the intermediate stimuli.
Participants and procedure. Twenty students at the University of Liverpool participated. The equipment and procedure were identical to the ones used in Experiment 1a.

\section{Results and Discussion}

We ran a repeated measures ANOVA using condition $(200 \mathrm{~cm}$, $176 \%$ magnification, or $500 \mathrm{~cm}$ ) as the within-subject variable. 
This confirmed an effect of condition, $F(2,38)=14.06, p<.001$. The mean response was -0.08 for the $200-\mathrm{cm}$ condition, -0.11 for the $176 \%$ magnification condition, and 0.053 for the $500-\mathrm{cm}$ condition. As in Experiment 1a, we also ran an item analysis with respect to the rooms and with the same independent variables as in the first ANOVA. The analysis confirmed an effect of condition, $F(2,12)=13.94, p<.001$.

As before, we tested the means against the value of zero. One-sample $t$ tests confirmed that the mean response for both the 200-cm condition, $t(19)=-3.39, p<.001$, and $176 \%$ magnification condition, $t(19)=-6.31, p<.001$, were significantly different from zero in the negative direction. The mean response for the 500-cm condition did not differ significantly from zero, $t(19)=1.26, p=.22$.

Figure 6 shows mean responses for the different conditions. Experiment $1 \mathrm{~b}$ demonstrated that both the magnification and the distance manipulations led to $\mathrm{BE}$, even when these trials were interleaved. The size of the BE effect was smaller for the magnification stimuli. This suggests that moving the vantage point was the most effective way to induce a perception of a close-up view. However, we need to be cautious, as the two manipulations were different in nature. Experiments $1 \mathrm{a}$ and $1 \mathrm{~b}$ together also confirm that $\mathrm{BE}$ only takes place for close-up images of a scene.

\section{Experiment 2: The Effect of Clutter Surrounding the Target Object}

There are two factors common to both magnification and reduced distance. One is the fact that fewer objects are visible around the target object, or in other words, the target object is set against a more homogeneous background. The second factor is the increase in retinal size of the target object. Experiment 2 and the subsequent experiments (in particular Experiments 4 and 5) test the role of these two factors. In these experiments we manipulated only the distance of the vantage point and not the magnification.

On the basis of the evidence in the literature, and in particular what was found in Intraub et al. (1992), clutter in the room should not be necessary for BE. Experiment 2 directly tests how, keeping everything else the same, the absence of furniture affects BE. If the homogeneous background is a factor, it is possible that a significant BE might emerge also at larger distances. Examples of stimuli are shown in Figure 7.

\section{Method}

Design and materials. The stimuli were similar to the stimuli used in Experiment 1a, with the exception that all furniture, except for the target objects, was removed from the seven rooms. The effect of the manipulation can be seen in Figure 7 for one of the rooms (Bedroom 1).

Participants and procedure. Twenty students at the University of Liverpool participated. The equipment and procedure were identical to those used in Experiment 1a.

\section{Results and Discussion}

A repeated measures ANOVA on distance $(200,350$, or $500 \mathrm{~cm}$ ) confirmed an effect of this variable, $F(2,38)=16.79, p<.001$. An item analysis with respect to the rooms and with the same independent variable as in the first ANOVA confirmed the effect of distance, $F(2,12)=65.19, p<.001$. The mean response was
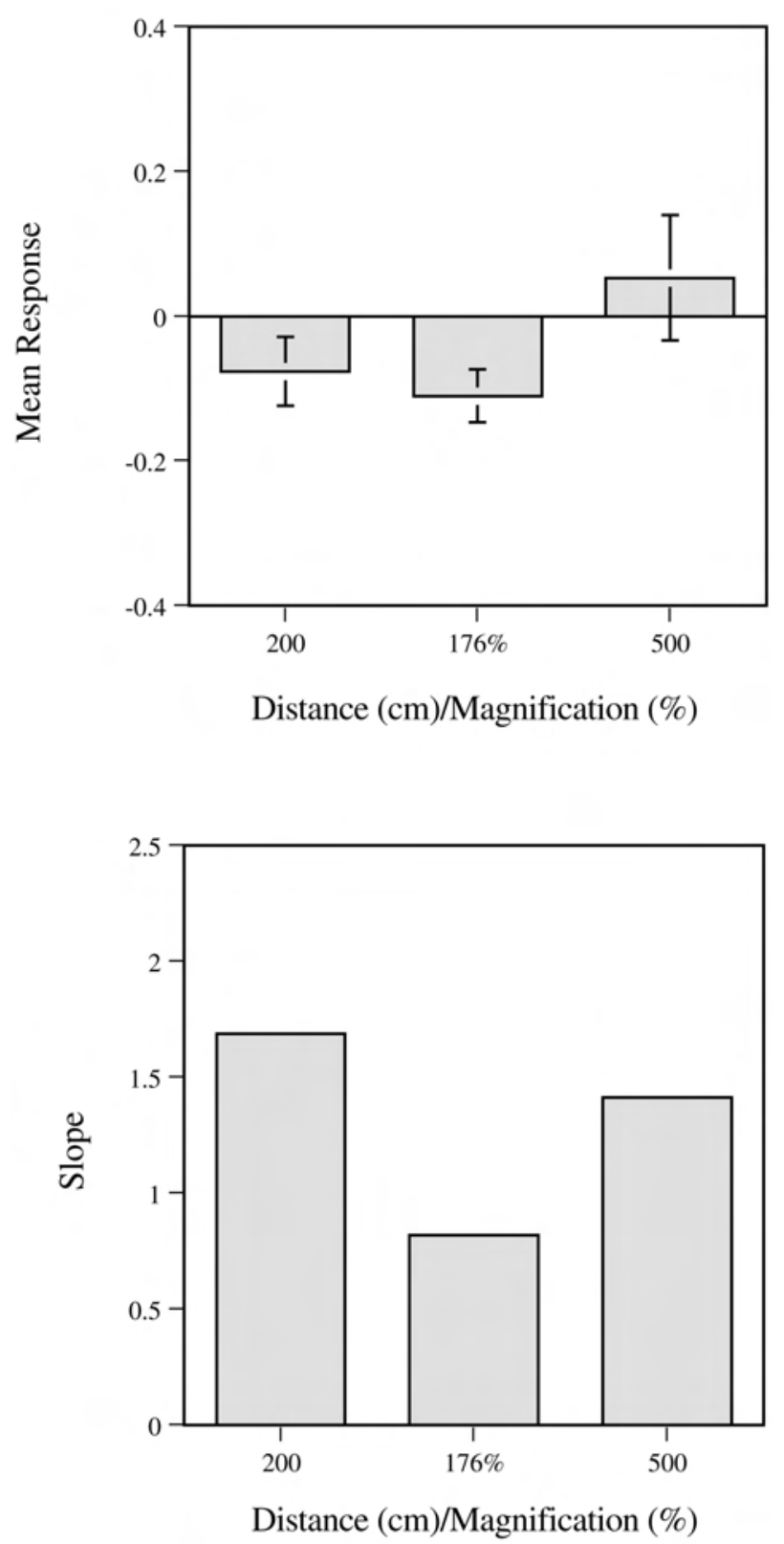

Figure 6. Data for Experiment 1b. Top: Overall mean responses for the three conditions. Error bars are 95\% confidence intervals. Bottom: Slopes for the three conditions.

-0.11 for the $200-\mathrm{cm}$ condition, 0.03 for the $350-\mathrm{cm}$ condition, and 0.21 for the $500-\mathrm{cm}$ condition.

One-sample $t$ tests confirmed that the mean response for the 200-cm condition, $t(19)=-2.63, p<.05$, was significantly different from zero in the negative direction. The $350-\mathrm{cm}$ condition was not significantly different from zero, $t(19)=0.68, p=.50$, and the $500-\mathrm{cm}$ condition was significantly different from zero in the positive direction indicating boundary contraction, $t(19)=$ $4.59, p<.001$. Figure 8 shows mean responses for the different distance conditions.

Unlike Experiments 1a and $1 \mathrm{~b}$, there is now clear evidence of a normalization process. There are two possible types of normaliza- 


\section{Experiment 2: Empty room}

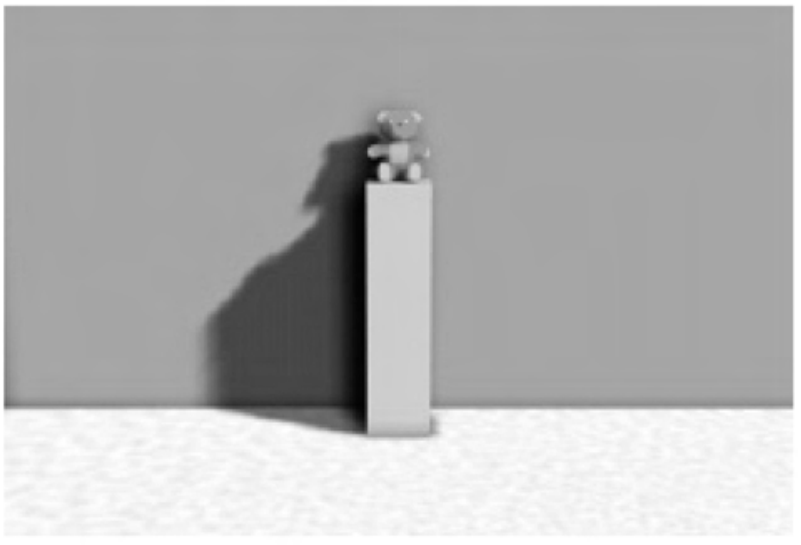

$500 \mathrm{~cm}$

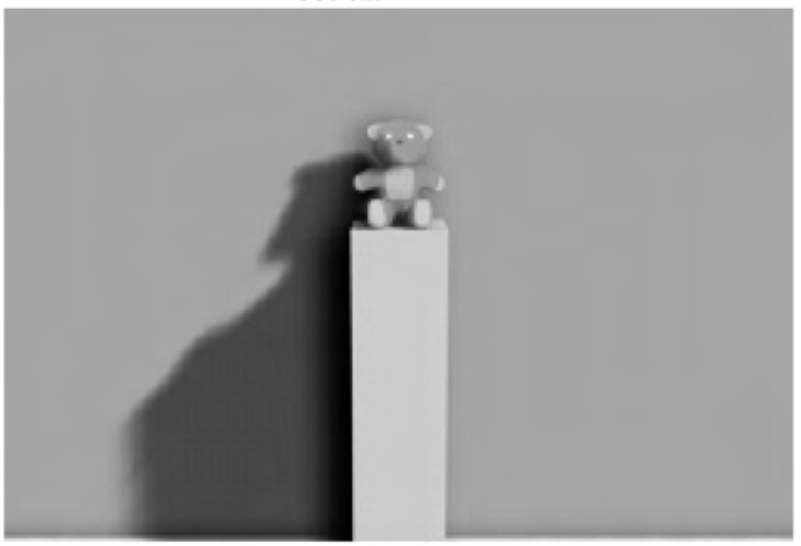

$350 \mathrm{~cm}$

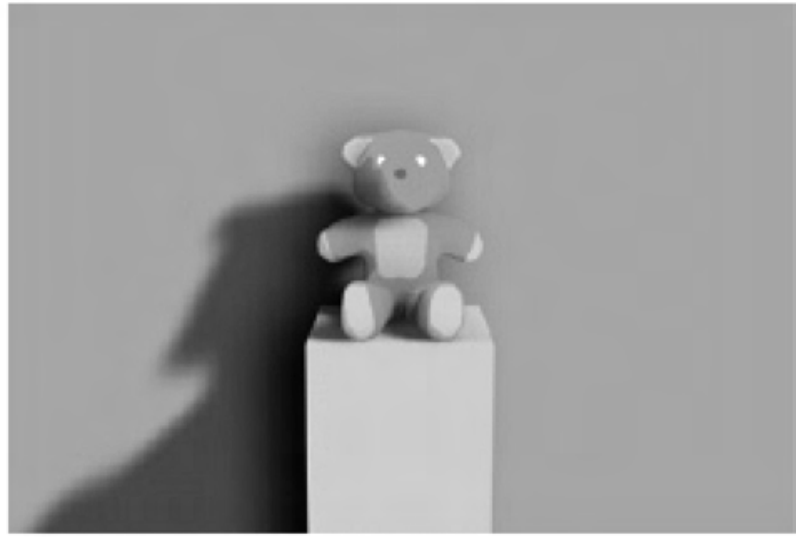

$200 \mathrm{~cm}$

Figure 7. Stimuli used in Experiment 2. Bedroom 1 is used as an example and can be compared with the stimuli shown in Figure 3.

tion relevant for BE. (a) The first is with respect to some default distance and could affect any experiment on BE. It has been argued that this is unlikely given that boundary contraction has not been reported for faraway views. A particular interpretation of this normalization process may account for that by assuming that when the view is closer than a preferred default, then observers remem- ber more than they saw; in other words, the effect of the default is asymmetric. This type of argument, however, moves in the direction of the perceptual schema hypothesis, and it overlaps with it to some degree. (b) Because all three distances were presented to each observer, in our experiments it is also possible that there was a within-experiment normalization process. In other words, it is possible that the judgments were affected by the trace of a mean distance computed over many trials. In Experiments 1a and 1b, we dismissed this possibility because there was no evidence of boundary contraction, but in Experiment 2 we saw evidence of this type of normalization. We believe that the explanation is that the
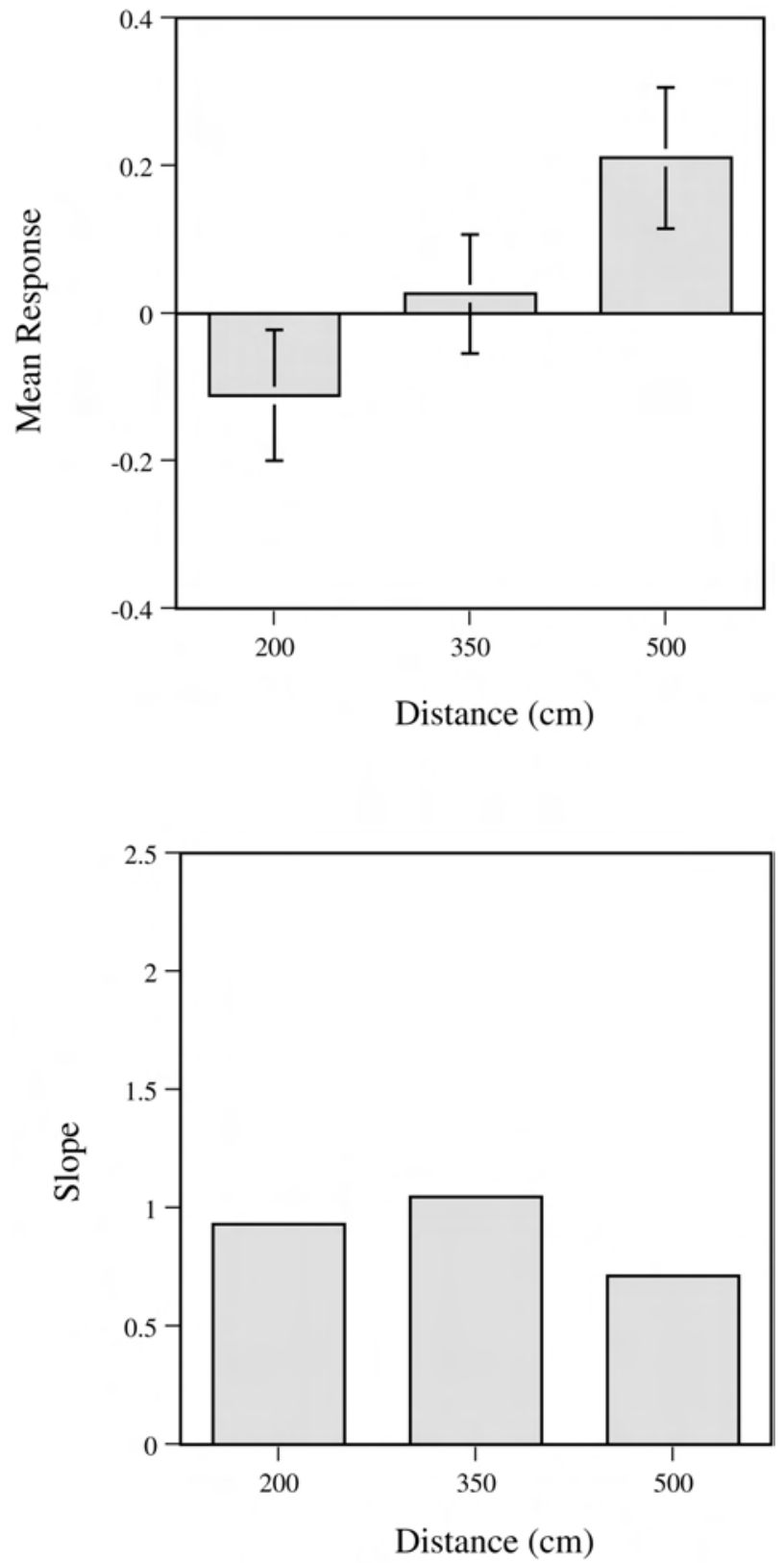

Figure 8. Data for Experiment 2. Top: Overall mean responses for the three conditions. Error bars are 95\% confidence intervals. Bottom: Slopes for the three conditions. 
removal of the furniture made the task much harder. Overall, in Experiment 2 slopes were much shallower than in Experiments 1a and $1 \mathrm{~b}$ (see Figure 8). Therefore we conclude that a shift toward the mean distance is likely to happen under condition of high uncertainty. It is important to stress that there was no evidence of anything of this kind happening in Experiments $1 \mathrm{a}$ and $1 \mathrm{~b}$. In particular, Figures 4 and 5 show that decreasing distance and increasing magnification (right to left) had opposite effects on task difficulty (slopes) and yet had the same effect on BE (present only for close-up views).

We conclude that clutter is not necessary but may be useful in testing BE. Without clutter, the image is more ambiguous, and the data seem better explained by normalization, that is, BE, but also boundary contraction. The issue of within-experiment normalization is further discussed in the next control experiment (Experiment 3).

\section{Experiment 3: Control: BE Without Normalization}

Experiment 2 suggested that our within-subject manipulation of distance may have led to some normalization in memory. Particularly when the task was hard (as it was without furniture) and less information was available to constrain the perceived distance (again because of the dearth of furniture), both BE and boundary contraction could be seen. We argued that this cannot explain the results of Experiment 1a on the ground that there was no relationship in that experiment between task difficulty and BE. Nevertheless, the normalization issue remains an important one. Experiment 3 is therefore a control in which a new group of participants only saw the close-up images of Experiment 1a (with furniture) and Experiment 2 (without furniture). If $\mathrm{BE}$ in Experiment 1a was exclusively a product of a normalization across trials, then neither BE nor boundary contraction should be present in Experiment 3 .

\section{Method}

Design and materials. The stimuli were taken from the 200-cm conditions of Experiment 1a (with furniture) and Experiment 2 (without furniture) and presented to naive observers. The rooms with furniture and those without furniture were presented to all participants.

Participants and procedure. Twenty students at the University of Liverpool participated. The equipment and procedure are described in the General Method section.

\section{Results and Discussion}

Figure 9 shows mean responses for different conditions. We ran a repeated measures ANOVA, with condition (furnished or unfurnished room) as a within-subject variable. The analysis revealed no effect of condition, $F(1,19)=0.196, p=.66$. An item analysis with respect to the rooms and with the same independent variables as in the first ANOVA confirmed no effect of condition, $F(1,6)=$ $0.23, p=.65$.

As there was no effect of condition, we collapsed the results across this variable for the $t$-test analysis. The mean response was -0.06 ; a one-sample $t$ tests confirmed that the mean response was significantly different from zero in the negative direction, $t(39)=$ $-2.35, p<.05$, consistent with BE. Experiment 3 confirms that

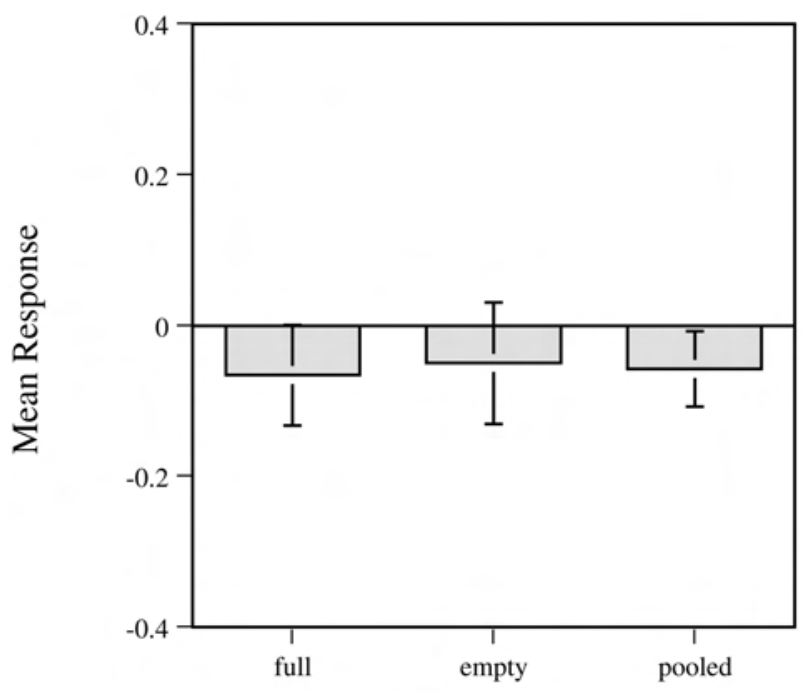

Condition

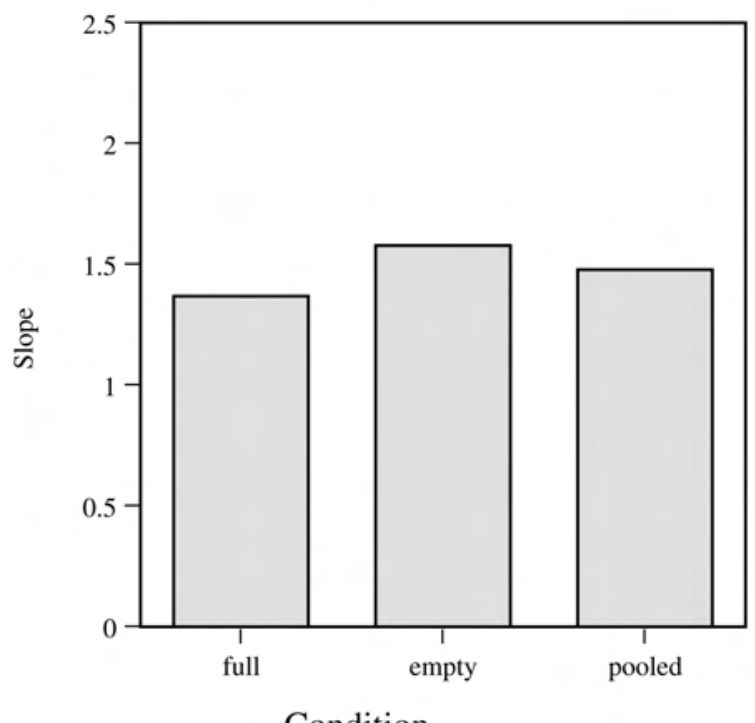

Condition

Figure 9. Data for Experiment 3. Top: Overall mean responses for the two conditions. Error bars are $95 \%$ confidence intervals. Bottom: Slopes for the two conditions.

BE can be found even when only close-up views are seen by the participants, therefore a within-experiment normalization cannot completely explain BE.

\section{Experiment 4: The Role of Target-Size Large Objects}

In our first experiment we matched the increase in size so that at $200 \mathrm{~cm}$ and at the corresponding magnification level (176\%) the target occupied the same percentage of the vertical dimension of the photograph. Perhaps this increase in size of the target object is a critical factor. In Experiment 4 we used the same seven rooms that we have used before, we included the $500-\mathrm{cm}$ and the $200-\mathrm{cm}$ 
distances as baseline conditions, and we introduced a new condition in which the object was larger. There are two ways of doing this, and we decided to adopt both. The first way is to use the same target object but rescale it to make it taller. These taller objects at a distance of $500 \mathrm{~cm}$ from the vantage point occupied exactly the same amount of the image as the shorter objects at $200 \mathrm{~cm}$.
Another way to describe this experiment is to consider the $500-\mathrm{cm}$ condition and two manipulations starting from it, a change in distance and a change in object size, both leading to the same retinal size of the object in the image (see Figure 10). The second way to have large objects at a distance of $500 \mathrm{~cm}$ is to use new objects. We chose them so that when they were seen from $500 \mathrm{~cm}$

\section{Large object (same)}

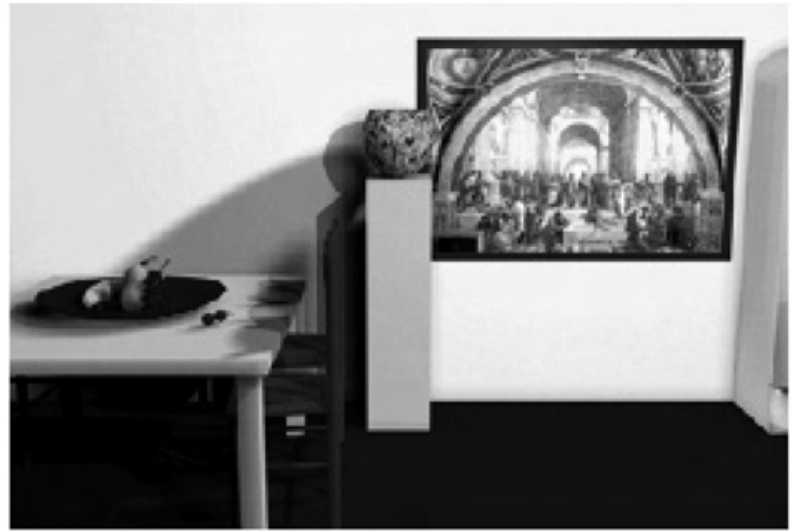

$500 \mathrm{~cm}$

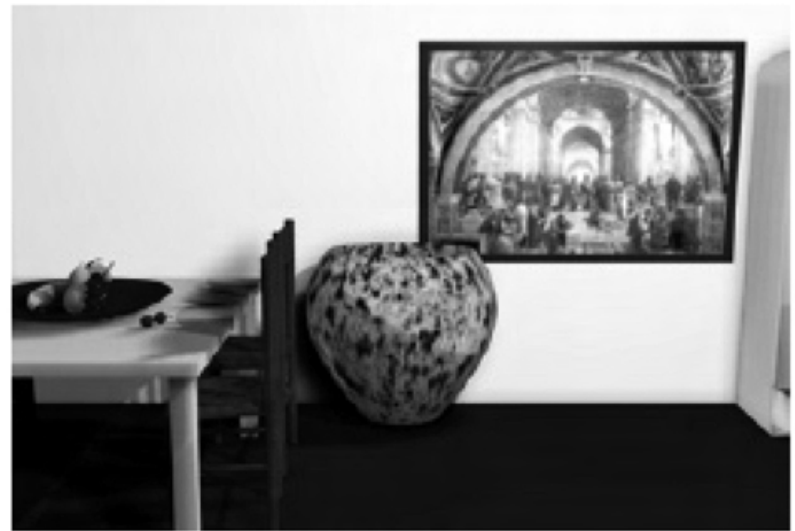

Large object

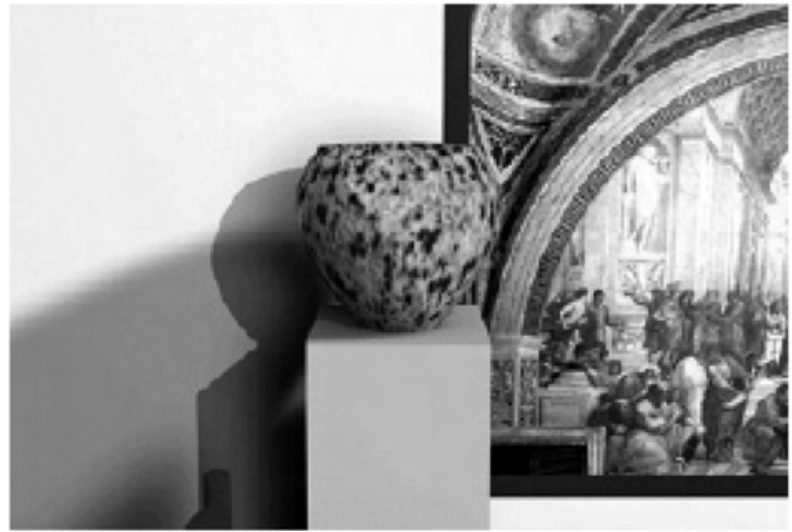

$200 \mathrm{~cm}$

\section{Large object (new)}

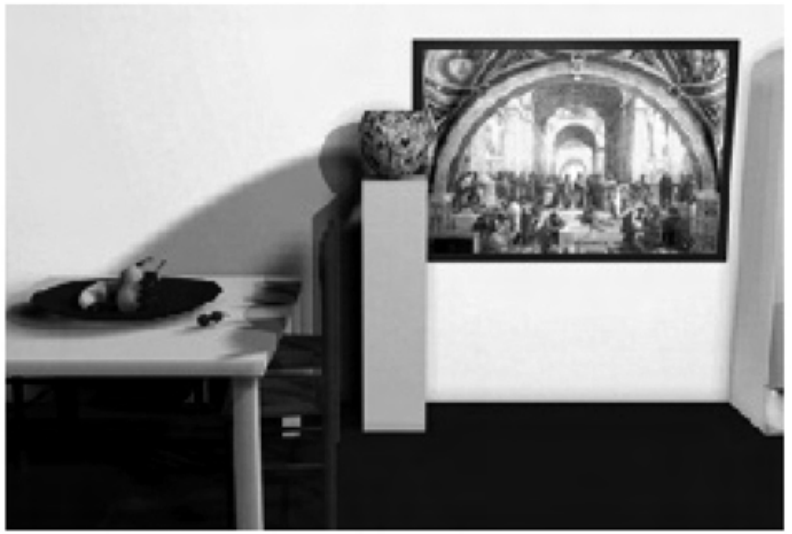

$500 \mathrm{~cm}$

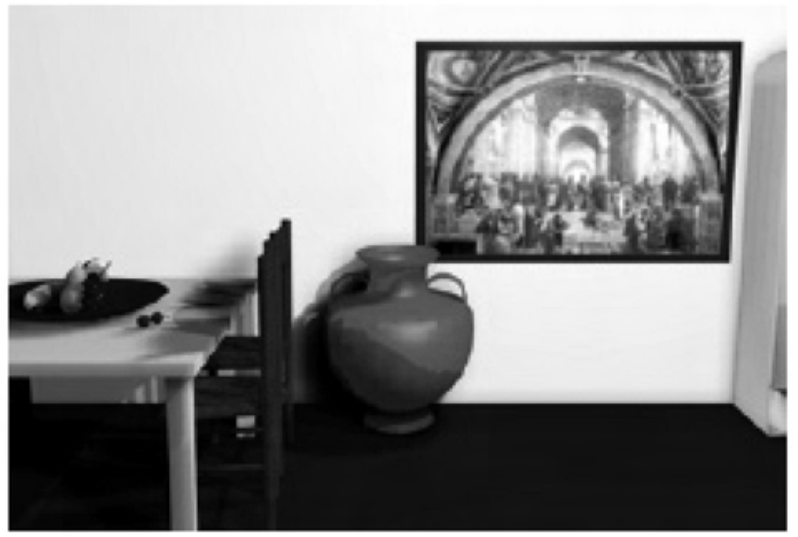

New object

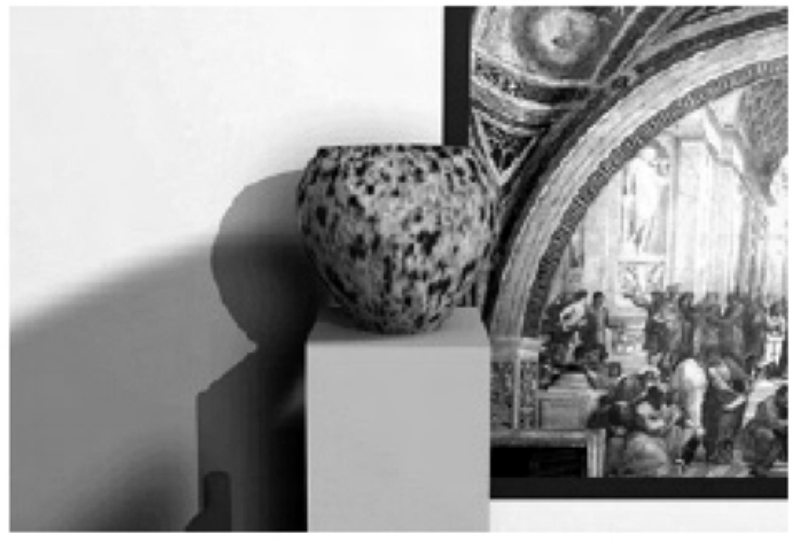

$200 \mathrm{~cm}$

Figure 10. Stimuli used in Experiment 4. The dining room is used as an example. Note that the stimuli in the top and bottom row are identical; the critical condition is the middle row. 
they occupied exactly the same amount of the image as the shorter objects at $200 \mathrm{~cm}$ (see Figure 10).

The first manipulation has the advantage that the nature of the object is unchanged. The second manipulation has the advantage that it avoids the problem of observers noticing a given object changing absolute size from trial to trial, for instance, a small teddy bear turning into a large teddy bear. If image size of target is important, we predict a significant BE not only at $200 \mathrm{~cm}$ but also at $500 \mathrm{~cm}$ for the taller objects (of both kinds).

\section{Method}

Design and materials. The stimuli for the 500-cm and $200-\mathrm{cm}$ conditions were taken from Experiment 1 and mixed with a new condition in which the vantage point was at $500 \mathrm{~cm}$ but the object was rescaled by $36 \%$ or replaced by a new target object. The new objects were a flower pot, a bedside cabinet, a washing machine, an amphora, a chair, a filing cabinet, and a telescope. The two manipulations were presented to two groups of participants.

Participants and procedure. Thirty students at the University of Liverpool participated. The equipment and procedure are described in the General Method section. Half of the participants saw the version of the experiment in which the object was rescaled, and the other half the version in which it was replaced by a different target.

\section{Results and Discussion}

We ran a repeated measures ANOVA, with condition (200-cm normal-sized object, 500-cm large object, 500-cm normal-sized object) as a within-subject variable and object type (same or different) as a between-subjects variable. The analysis confirmed an effect of condition, $F(2,56)=20.09, p<.001$, and no effect of object type, $F(1,28)=2.11, p=.16$, or interaction between the two, $F(2,56)=1.91, p=.16$. An item analysis with respect to the rooms and with the same independent variables as in the first ANOVA confirmed an effect of condition, $F(2,24)=12.22, p<$ .001 , and no effect of object type, $F(1,12)=1.03, p=.33$, or interaction between the two, $F(2,24)=1.21, p=.32$.

As there was no effect of object, we collapsed the results across this variable for the $t$-test analysis. The mean response was -0.13 for the 200-cm normal-sized object condition, -0.08 for the $500-\mathrm{cm}$ large object condition, and 0.03 for the $500-\mathrm{cm}$ normalsize object condition. One-sample $t$ tests confirmed that the mean response for both the 200-cm condition, $t(29)=-5.64, p<.001$, and 500-cm large object condition, $t(29)=-3.83, p<.01$, were significantly different from zero in the negative direction, as predicted by BE. The mean response for the 500-cm normal-sized object was not significantly different from zero. Mean responses for the different conditions are shown in Figure 11.

We did not expect the size of the target object to be a critical factor, but from the results we must accept that it plays a significant role, although this is not to say that it is the only factor. Familiarity with the size of the object was not important because there was no difference between the two manipulations (same and new objects). Perhaps an even more interesting finding from this experiment is that $\mathrm{BE}$ cannot be understood by concentrating entirely on what happens at the boundary of the image. As can be seen in Figure 10, the 500-cm conditions with the original and with
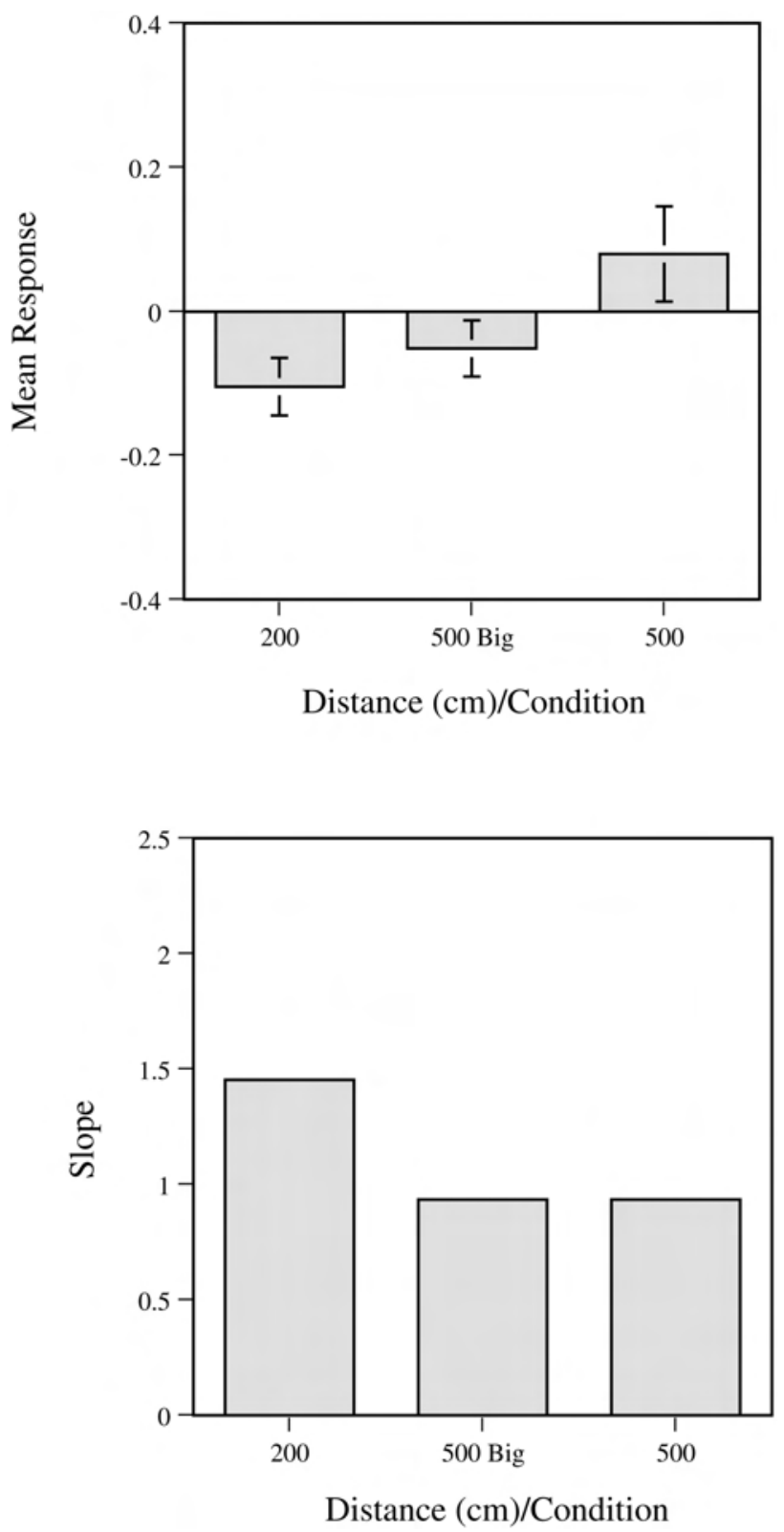

Figure 11. Data for Experiment 4. Top: Overall mean responses for the three conditions. Error bars are 95\% confidence intervals. Bottom: Slopes for the three conditions.

the larger object are identical in terms of the information at the boundaries. Yet the responses to these two conditions were significantly different.

\section{Experiment 5: The Role of Target-Size Small Objects}

Against our expectations, in Experiment 4 we found that retinal size of the target object was a significant factor. We believe this is by no means the only factor; nevertheless, to better understand the role of object size we wanted to know what happens when we use a small object for a close-up view (200-cm distance). Experiment 5 complements Experiment 4 in that the small objects were scaled 
to have the same retinal size as the large objects at the larger distance. Similar to what was done in Experiment 4, we presented both rescaled objects and new small objects (see Figure 12).

\section{Method}

Design and materials. The stimuli for the $500-\mathrm{cm}$ and $200-\mathrm{cm}$ conditions were taken from Experiment 1 and mixed with a new condition in which the vantage point was at $500 \mathrm{~cm}$ but the object was rescaled by $36 \%$ or replaced by a new target object. The new objects were a pear, a mug, a wine glass, an apple, a chess piece, a stapler, and an hourglass. The two manipulations were presented to two groups of participants.

Participants and procedure. Thirty students at the University of Liverpool participated. The equipment and procedure are described in the General Method section. Half of the participants saw the version of the

\section{Small object (same)}

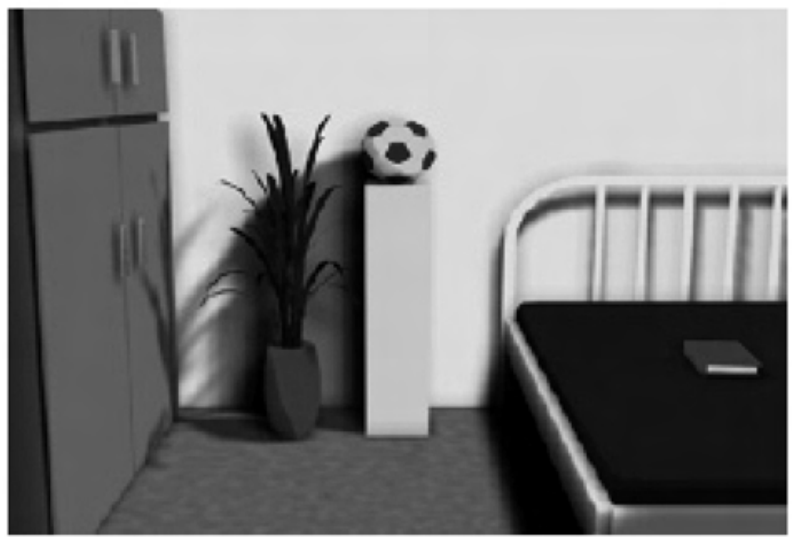

$500 \mathrm{~cm}$
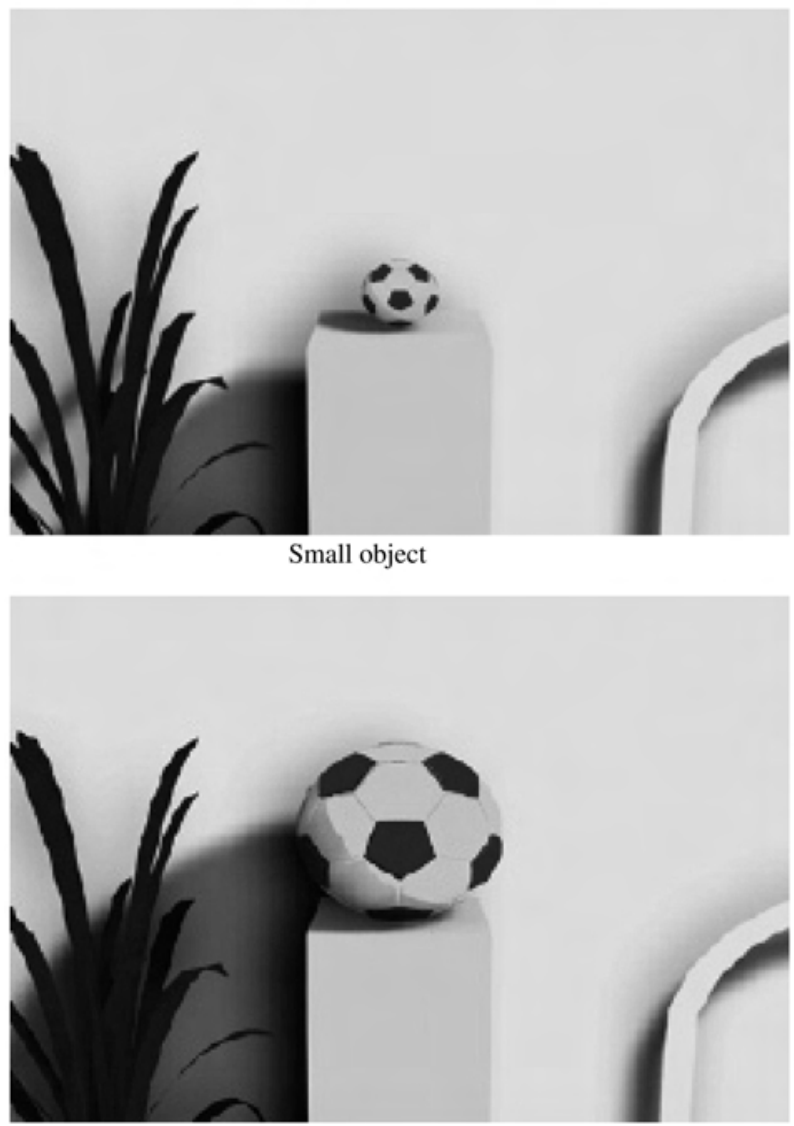

$200 \mathrm{~cm}$

\section{Small object (new)}

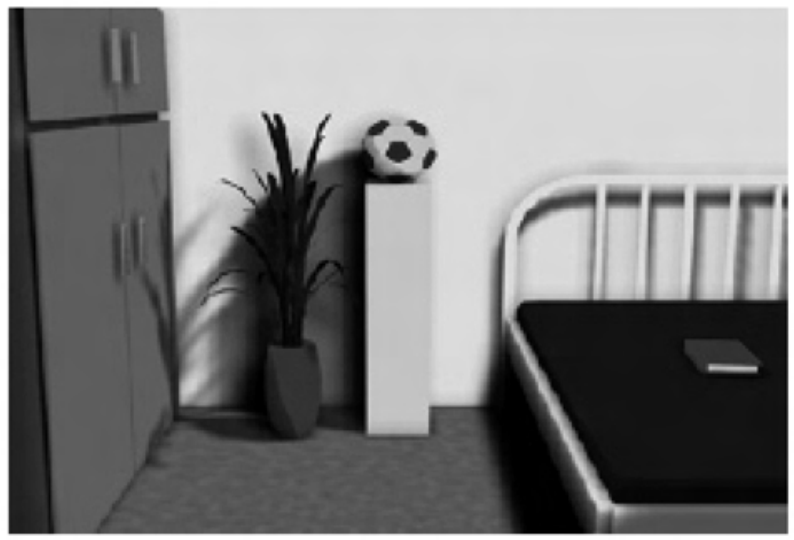

$500 \mathrm{~cm}$

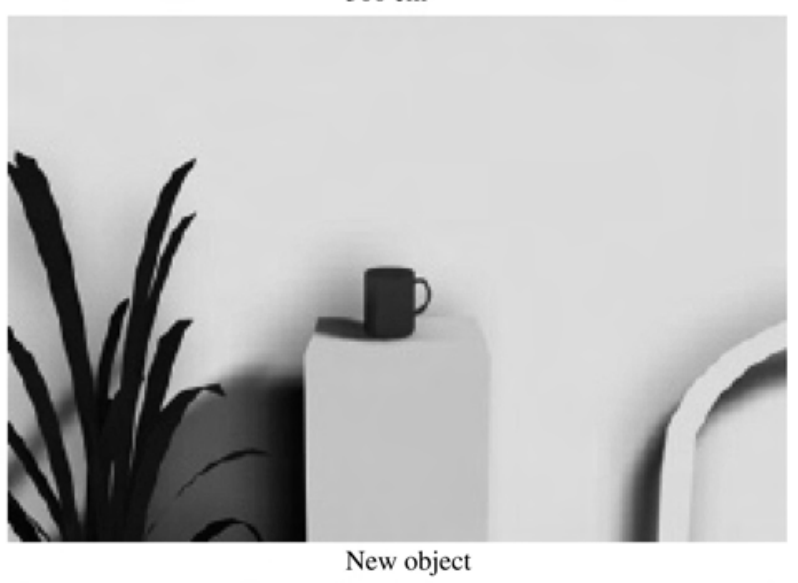

New object

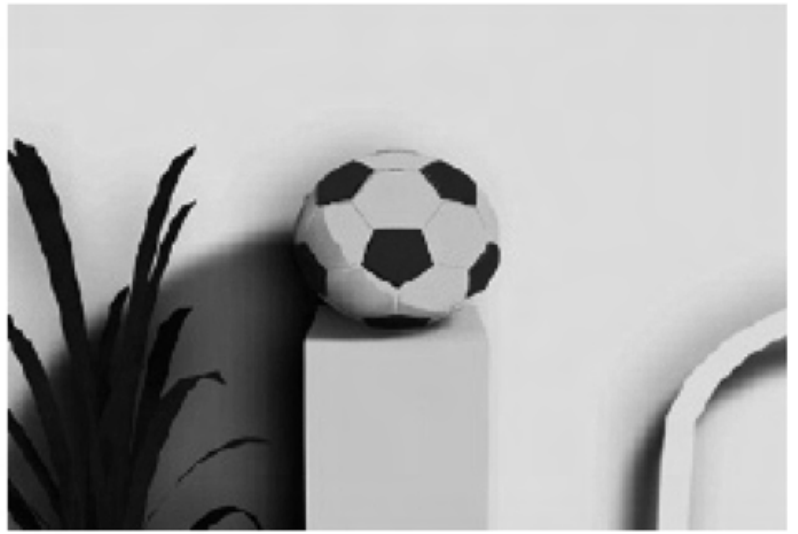

$200 \mathrm{~cm}$

Figure 12. Stimuli used in Experiment 5. Bedroom 2 is used as an example. 
experiment in which the object was rescaled, and the other half the version in which it was replaced by a different target.

\section{Results and Discussion}

Because of an error in the graphic display, the data from one of the seven items could not be used. The data from the other six items were analyzed. ${ }^{2}$ We ran a repeated measures ANOVA, with condition (200-cm normal-sized object, $200-\mathrm{cm}$ small object, $500-\mathrm{cm}$ normal-sized object) as a within-subject variable and object type (same or different) as a between-subjects variable. The analysis confirmed an effect of condition, $F(2,56)=8.57, p<$ .001 , and no effect of object type, $F(1,28)=0.42, p=.52$, or interaction between the two, $F(2,56)=0.38, p=.68$. An item analysis with respect to the rooms and with the same independent variables as in the first ANOVA confirmed an effect of condition, $F(2,30)=77.15, p<.001$, and no effect of object type, $F(2$, $15)=0.57, p=.577$, or interaction between the two, $F(2,30)=$ $1.56, p=.21$

As there was no effect of object type, we collapsed the results across this variable for the $t$-test analysis. The mean response was -0.22 for the $200-\mathrm{cm}$ condition, -0.05 for the $200-\mathrm{cm}$ smallobject condition, and 0.12 for the $500-\mathrm{cm}$ condition. One-sample $t$ tests confirmed that the mean response for the $200-\mathrm{cm}$ condition, $t(29)=-3.92, p<.001$, was significantly different from zero in the negative direction, as predicted by BE. The mean responses for both the $500-\mathrm{cm}$ normal-sized object and $200-\mathrm{cm}$ small-object condition were not significantly different from zero.

Figure 13 shows mean responses for different conditions. Experiment 5 confirms the finding from Experiment 4 that retinal size of the target object is important. Even at a distance of $200 \mathrm{~cm}, \mathrm{BE}$ can disappear when the object is small. Taken together, Experiments 4 and 5 strongly point to the conclusion that information at the boundaries of the picture is not the only type of information that affects BE.

\section{Experiment 6: Binocular and Monocular Viewing}

The manipulation in this experiment is simple but important. There are many reasons why pictures may be special; one of them is the conflicting information that we have from the lack of any binocular disparity for the objects in the scene. For pictures there is no difference between how much of the scene is seen by either eye. This is different from what happens when looking at a scene through a window or aperture. One reason this is interesting is that such conflicting information should be greater when the boundaries are perceived as near the objects in the scene, and perhaps this is why BE requires scenes to be perceived as close-up.

Another reason why monocular viewing is interesting is the fact that it has long been recognized as a way to enhance the depth perception in photographs (Ames, 1925; Schlosberg, 1941). In the introduction we explained that we are particularly interested in perception of space, and that is why we started investigating BE. If $\mathrm{BE}$ is directly related to perception of space, then the increased sense of depth from monocular viewing might increase the $\mathrm{BE}$ effect.

\section{Method}

Design and materials. The stimuli for the $500-\mathrm{cm}$ and $200-\mathrm{cm}$ conditions were taken from Experiment 1. Half of the participants performed the
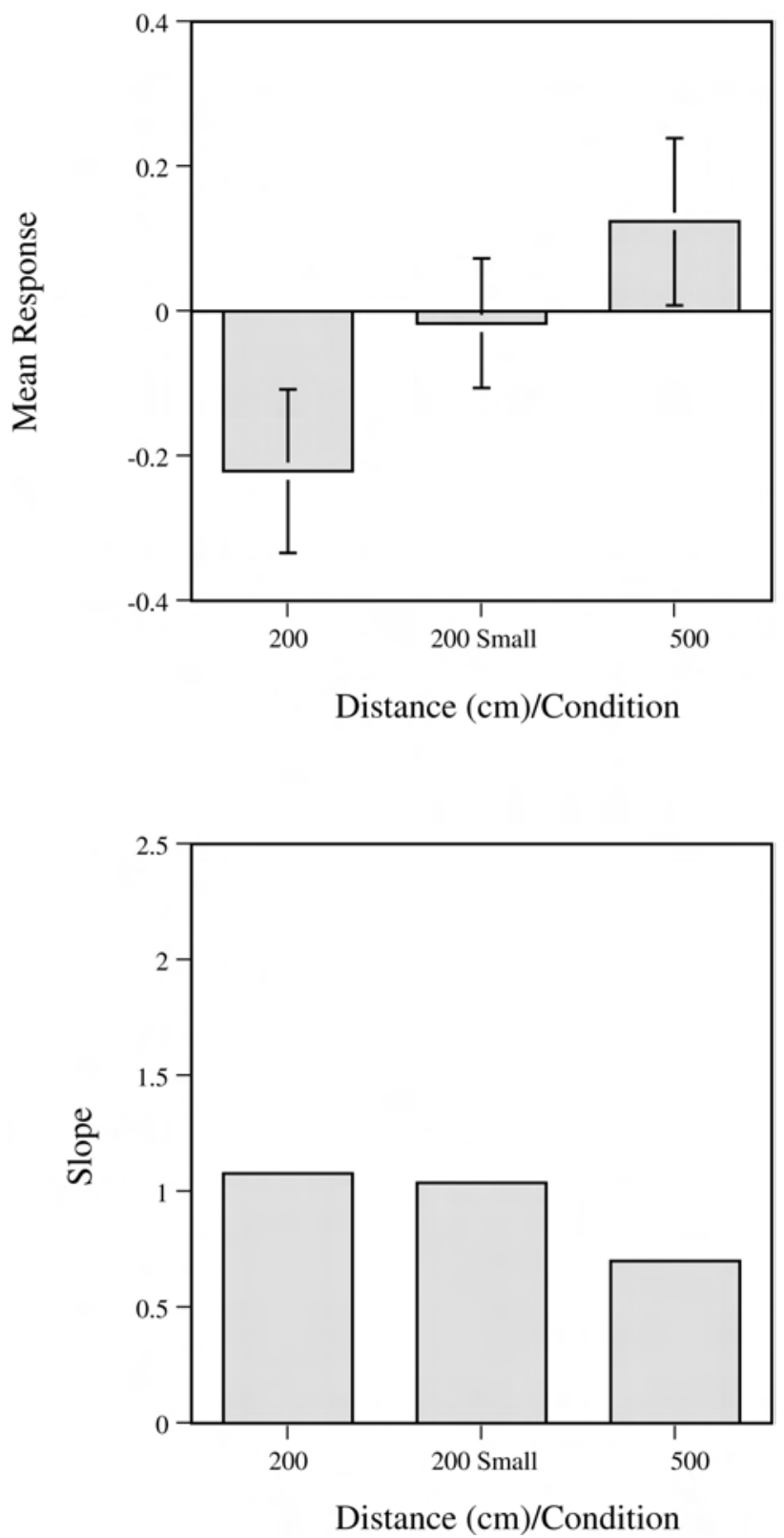

Figure 13. Data for Experiment 5. Top: Overall mean responses for the three conditions. Error bars are 95\% confidence intervals. Bottom: Slopes for the three conditions.

task binocularly and subsequently monocularly; the other half of the participants followed the reverse order. In the monocular trials, participants always used their dominant eye and wore an eye-patch on the nondominant eye.

\footnotetext{
${ }^{2}$ There was a mistake in the setting of the standard distance for one of the rooms; this was a vertical error of $10 \mathrm{~cm}$ (i.e., vantage point higher than for the other rooms). We also performed an analysis with all seven rooms, and the results were fundamentally identical to those reported for the six rooms.
} 
Participants and procedure. Twenty-four students at the University of Liverpool participated. The equipment and procedure are described in the General Method section.

\section{Results and Discussion}

We ran a repeated measures ANOVA, with distance $(200 \mathrm{~cm}$ and $500 \mathrm{~cm}$ ) and viewing condition (binocular and monocular) as within-subject variables and order (binocular first or vice versa) as a between-subjects variable. The analysis confirmed a nonsignificant effect of distance, $F(1,22)=2.39, p<.1363$; a significant effect of viewing, $F(1,22)=8.91, p<.01$; but no effect of order, $F(1,22)=1.74, p=.20$. There were no significant interaction effects. We ran an item analysis with respect to the rooms and with the same independent variables as in the first ANOVA. The analysis confirmed a significant effect of distance, $F(1,6)=$ $86.54, p<.001$; a significant effect of viewing, $F(1,6)=10.19$, $p<.05$; but no interaction between the two, $F(1,6)=0.54, p=$ .49 .

The mean response was -0.04 for the monocular $200-\mathrm{cm}$ condition, 0.02 for the monocular $500-\mathrm{cm}$ condition, -0.20 for the binocular $200-\mathrm{cm}$ condition, and -0.02 for the binocular $500-\mathrm{cm}$ condition. Figure 14 shows mean responses for the different conditions.

One-sample $t$ tests confirmed that the mean response for the binocular 200-cm condition, $t(23)=-4.71, p<.001$, was significantly different from zero in the negative direction. However, the mean response for the monocular $200-\mathrm{cm}$ condition, $t(23)=$ $-0.76, p=.46$, was not significantly different from zero. The mean responses for both the monocular 500-cm condition, $t(23)=$ $0.31, p=.76$, and binocular 500-cm condition, $t(23)=-0.40$, $p=.69$, did not differ significantly from zero.

The outcome of Experiment 6 was remarkably clear-cut although surprising. BE was greater for binocular viewing compared with monocular viewing. ${ }^{3}$

\section{Experiment 7: Stereograms}

This experiment follows on directly from what was found in Experiment 6 and tests the importance of perceived depth using stimuli in which depth is explicitly specified by binocular disparity. In Experiment 7, we present the rooms in each trial as a pair of stereograms, interleaved with a control condition in which the same rooms are presented as pictures (zero disparity). Similar to the monocular viewing manipulation, there are two possibilities; the enhanced sense of depth may lead to an increase in BE, but if $\mathrm{BE}$ is a pictorial phenomenon it will be absent or reduced for stereograms, perhaps because no binocular conflict is present.

\section{Method}

Design and materials. The stimuli were similar to the $500-\mathrm{cm}$ and 200-cm conditions in Experiment 1. What was new in Experiment 7 was that pairs of stereograms were generated by taking virtual photographs of the rooms from two slightly different vantage points. Specifically, we used the average human interocular distance $(6.5 \mathrm{~cm})$. These stereograms were presented on a Sony F500T9 monitor with a resolution of 1,280 pixels $\times$ 1,024 pixels at $120 \mathrm{~Hz}$. Two stereo images were presented with the use of a NuVision infrared emitter and stereoscopic glasses. The effect of interleaving left and right images was that effective vertical resolution and

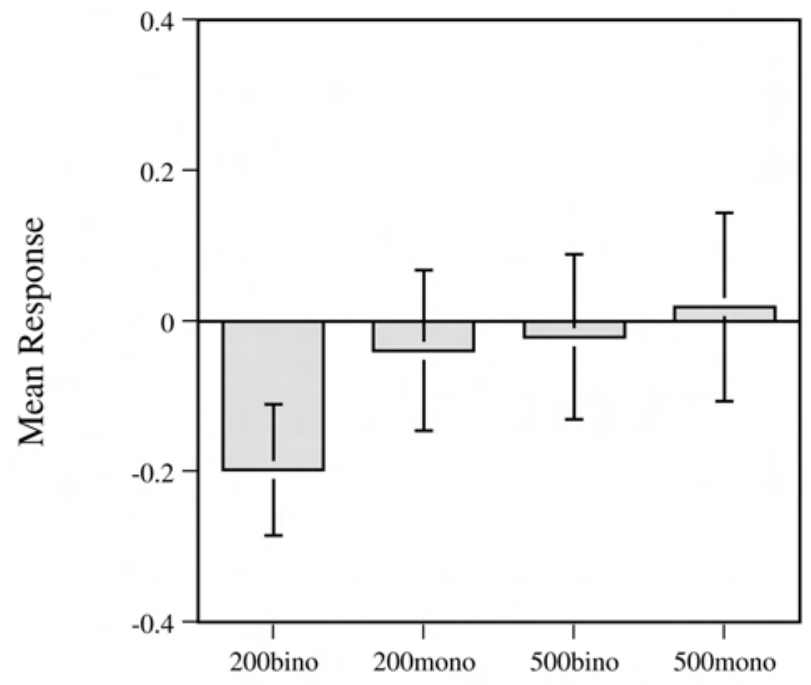

Distance $(\mathrm{cm}) /$ Condition

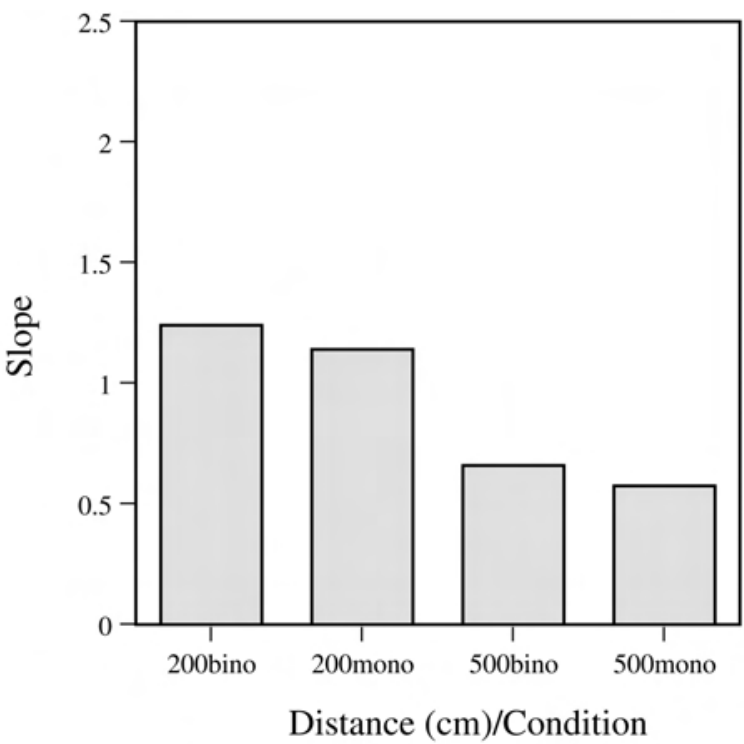

Figure 14. Data for Experiment 6. Top: Overall mean responses for the two conditions. Error bars are $95 \%$ confidence intervals. Bottom: Slopes for the two conditions. bino $=$ binocular condition; mono $=$ monocular condition.

refresh rate were halved $(512$ pixels at $60 \mathrm{~Hz})$. For technical reasons, we could only include four rooms in this experiment.

The following factors were factorially combined: virtual distance (500 and $200 \mathrm{~cm}$ ), change $(-15,0$, and $15 \mathrm{~cm})$, room (four different rooms), and

\footnotetext{
${ }^{3}$ The results from this particular experiment suggest that no $\mathrm{BE}$ is present for monocular viewing. However, we have replicated this experiment with a few modifications in our laboratory, and we believe that the correct conclusion is that monocular viewing reduces $\mathrm{BE}$ in a direct comparison with a binocular condition but does not completely eliminate BE.
} 
type (stereograms and nonstereograms). In the case of nonstereograms, only one of the two images from a stereogram was presented to both eyes.

Participants and procedure. Twenty students at the University of Liverpool participated. They were screened for normal stereo vision using the TNO (1972) stereo test. The procedure is described in the General Method section, but for this experiment the participants wore a pair of stereoscopic glasses throughout.

\section{Results and Discussion}

We ran a repeated measures ANOVA, with distance $(200 \mathrm{~cm}$ and $500 \mathrm{~cm}$ ) and type of images (stereograms and pictures) as within-subject variables. The effect of type of images was not significant, $F(1,19)=0.05, p=.823$, and there was no interaction between type and distance, $F(1,19)=0.41, p=.528$. The variable distance did not reach significance, $F(1,19)=3.34, p<.083$.

For the stereograms, the mean response was -0.16 for the $200-\mathrm{cm}$ condition and -0.03 for the $500-\mathrm{cm}$ condition. For the images presented without disparity, the mean response was -0.13 for the $200-\mathrm{cm}$ condition and -0.04 for the $500-\mathrm{cm}$ condition. Figure 15 shows mean responses for the different conditions.

Even though the ANOVA did not show a difference when the $200-\mathrm{cm}$ and the 500-cm conditions were compared, they did differ in their overlap with zero. One-sample $t$ tests confirmed that the mean response for the 200-cm condition, $t(19)=-2.75, p<.013$, was significantly different from zero in the negative direction. However, the mean response for the $500-\mathrm{cm}$ condition, $t(19)=$ $-0.94, p=.36$, was not significantly different from zero. There was a high degree of variability for the $500-\mathrm{cm}$ condition (see Figure 15). Perhaps this relates to something we have noted before, namely that if the objects look toylike the room may seem small and near, making this a close-up condition for some observers.

The outcome of Experiment 7 helps us in understanding the outcome of Experiment 6. It is not the case that the binocular conflict (in pictorial images) is necessary for finding BE. Together, Experiments 6 and 7 suggest that BE does not increase with perceived depth in the images.

\section{General Discussion}

In a series of experiments, we have found that $\mathrm{BE}$ takes place with computer-generated images of rooms using a new methodology in which the same room and the same view are presented several times during the experiment. Our new methodology adds generality and tests specific factors that could not be easily tested with real photographs; for instance, the role of object size while keeping everything else constant. We confirm that BE is a perceptual effect that has two prerequisites: the presence of an extended background and a close-up view of the scene to be remembered. Next, we discuss BE in relation to other literatures, and we draw conclusions from our own new findings.

\section{BE and Picture Perception}

There is a clear link between BE and other issues in the area of picture perception (Hagen, 1980; Kennedy, 1974). In many ways $\mathrm{BE}$ is eminently about pictures and the existence of boundaries that crop a scene. We are not aware of any equivalent bias in memory for a scene other than when pictures are involved, except for a study by Intraub (2004). In this study participants explored a
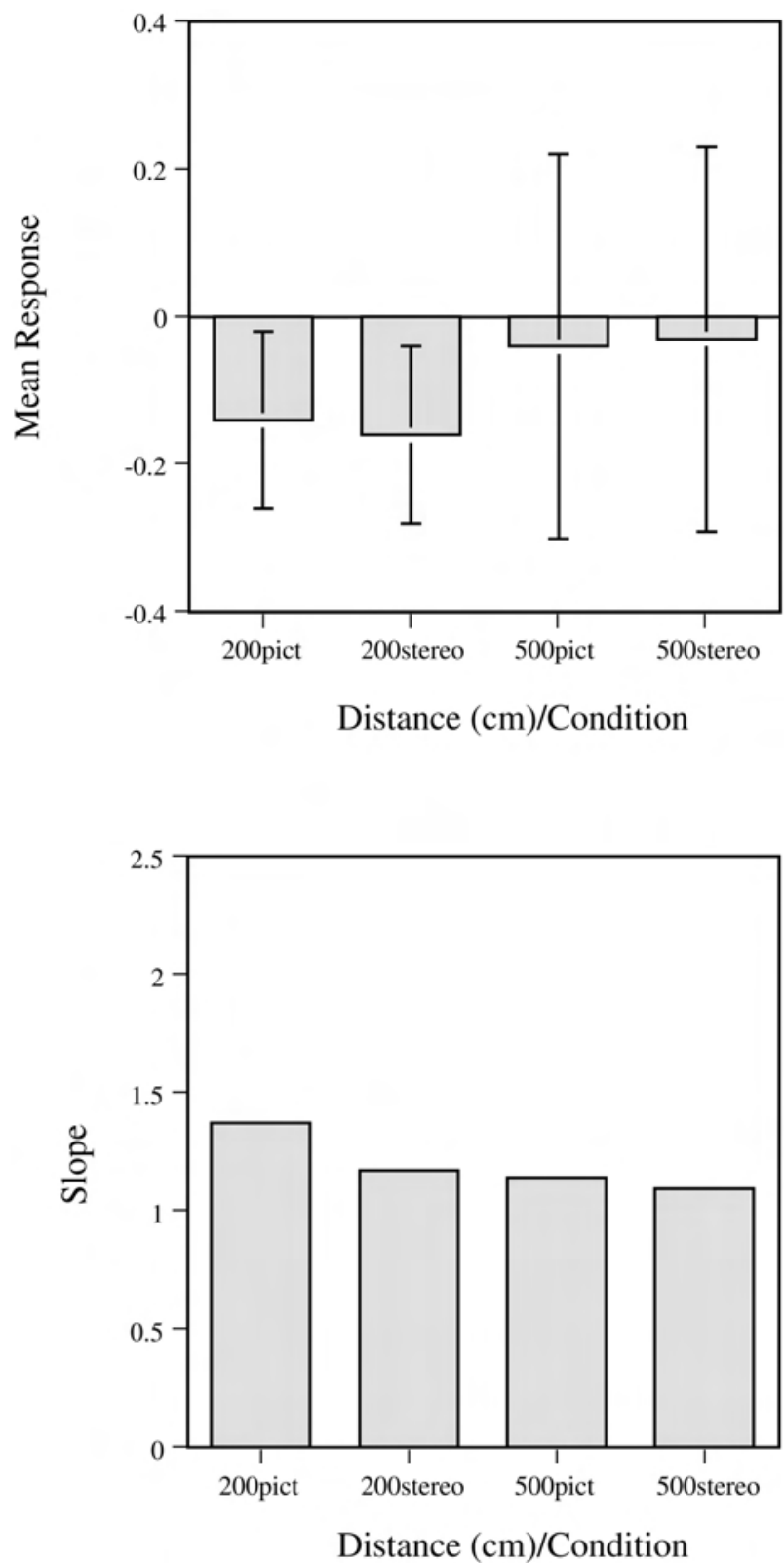

Figure 15. Data for Experiment 7. Top: Overall mean responses for the two conditions. Error bars are $95 \%$ confidence intervals. Bottom: Slopes for the two conditions. pict $=$ pictorial condition; stereo $=$ stereoscopic condition.

region of space on which a few objects (e.g., a doll or a brush) had been placed and were enclosed by a window. After visual or haptic exploration and a short delay, they set the size of the window and made the area significantly larger. This study is important and the effect clear cut; nevertheless, at present it is not clear whether it should be treated as exactly the same as the BE found in other studies. To know that it is the same phenomenon, it should display similar characteristics: be specific to scenes and not other spatial information, be stronger for close-up, and be present for recognition as well as reproduction. Conversely, BE for pictures should be 
tested to see what happens when a larger frame is presented at the time of testing, without changing the size of the objects. Future research will have to explore this phenomenon in more detail, and the relationship between data from different sensory modalities may be a complex one.

One important aspect of $\mathrm{BE}$ is that it is stronger for close-up views. Painters have long been aware that there is a range outside which images look distorted, and since Leonardo da Vinci the advice was to not exceed $45^{\circ}-60^{\circ}$ of visual angle horizontally. This is close to the horizontal field of view of a normal lens (i.e., a lens in the 45-50-mm range). But distortions also appear when the visual angle is too small, for instance, as a consequence of using a telephoto lens when taking the picture and later failing to adjust the viewing distance (Cutting, 2003; Hecht, van Doorn, \& Koenderink, 1999; Kubovy, 1986; Lumsden, 1980, 1983). A perceptual result of such use of a zoom lens is a depth compression, which for complex objects also leads to distortion of shape.

In Experiment 1, we tested the hypothesis that $\mathrm{BE}$ may originate in conditions in which a photograph was taken with a lens outside the normal range. In other words, a possible definition of a close-up is that it is a photograph taken with a zoom lens and therefore showing a limited horizontal field of view. If so, BE would be more an artifact than a general perceptual effect taking place when observers represent space as extending beyond the boundaries of the scene. We manipulated angle of view and distance separately to generate different "close-up" images and found a significant BE in both cases. Even more conclusive evidence that $\mathrm{BE}$ is not a mere picture perception phenomenon comes from Experiment 7, in which $\mathrm{BE}$ was present for stereograms.

\section{BE and Change Blindness}

Change blindness is a striking phenomenon and has generated great recent interest and debate (for a review, see Simons, 2000). As the name implies, change blindness is about the limits of memory for scenes. Across saccades, blank screens, or other interruptions, observers fail to detect substantial changes to the visual details of scenes. In contrast, $\mathrm{BE}$ is a systematic bias in one direction. One might argue that if memory for scenes is poor, observers may rely on contextual information about what is likely to be present in the scene. In the context of change blindness, it has been suggested that observers only extract the meaning or gist of a scene but not its full spatial details (Friedman, 1979). This parallel is appealing, but in itself it does not help to explain the direction of the BE effect. More extent of the background surface and smaller objects do not make the scene more meaningful in any obvious sense.

\section{$B E$ and Naive Physics}

Our own interest in BE originated from work in naive physics. Naive physics is the study of commonsense beliefs about simple physical phenomena (Bozzi, 1990; Proffitt, 1999). Naive beliefs are often found to be at odds with reality, for instance, when asked where to drop a ball to hit a target on the floor while moving in an airplane or on a conveyor belt (e.g., Kaiser, Proffitt, Whelan, \& Hecht, 1992; McCloskey, Washburn, \& Felch, 1983), many adults state that they should release the ball right above the target. Within naive physics, naive optics study beliefs about phenomena that depend on the laws of optics. One case relevant to our everyday life is the behavior of mirrors. Croucher, Bertamini, and Hecht (2002) and Bertamini, Spooner, and Hecht (2003) have found that adults tend to overestimate what is made visible by a planar mirror. It appears that observers find it difficult to judge what is visible in a mirror as well as what is visible from a given vantage point (Bertamini, Latto, \& Spooner, 2003; Bertamini \& Parks, 2005; Hecht, Bertamini, \& Gamer, 2005). There may be a few factors involved in this effect, one of them is the possibility that, just like for BE, observers represent the scene beyond the boundaries of a mirror. The fact that $\mathrm{BE}$ is strongest for close-up views is not a problem for this hypothesis because arguably most of our everyday experience with planar mirrors is from a relatively short distance. The finding that $\mathrm{BE}$ is not an artifact of magnification is also in agreement with such hypothesis, and so is the presence of BE for stereograms because, unlike a photograph, a real planar mirror always shows a scene from a vantage point consistent with all available information about distance (e.g., convergence and stereopsis).

\section{Summary of New Findings}

With respect to close-up views, Experiments 1a and 1b tested the hypothesis that BE is found specifically when a close-up is achieved by means of magnification, as present for instance when using a zoom lens. This was not the case.

We also confirmed that $\mathrm{BE}$ is not the product of the reduced clutter in the close-up views (Experiment 2), although empty rooms made the task harder, and in such conditions we found evidence of a normalization process. This process can lead to both a BE and a boundary contraction. We report slopes as a measure of performance for all our experiments. It is important to note that in all experiments for which performance was good, there was still evidence of $\mathrm{BE}$ but no evidence of contraction. Comparing the difficulty of close-up views and faraway views does not support the hypothesis that it is an increase in difficulty for close-up views that leads to the memory bias (see, for instance, Figure 3). As discussed in the introduction, the presence of extension but not contraction is relevant to favor the perceptual schema rather than the memory schema hypothesis.

Experiment 3 was another control experiment. Pictures were presented to observers without being interleaved with images taken from a farther vantage point. The results confirm that BE exists under these conditions, and it is not therefore entirely explained by normalization.

In Experiments 4 and 5, we tested whether BE depends on the presence of an object taking up a large proportion of the image. Unexpectedly, size of the target object had a significant impact, leading to $\mathrm{BE}$ for the $500-\mathrm{cm}$ condition in Experiment 4 and the elimination of BE for the 200-cm condition in Experiment 5. Note that apart from the change in the target object, these images were identical to the control images presented in the same experiment. Observers clearly were influenced by the change in the target object (see Figures 11 and 13). In other words, BE was present mostly when the target object was occupying a large portion of the scene. This finding is surprising because target size is a factor unrelated to the occlusion of the boundaries or the overall layout of the scene. It is possible that larger sizes for the target object 
contributed to the perception of close-up. If so, retinal size is a factor that acts indirectly.

The results from Experiment 6 and 7 were perhaps the most surprising. In Experiment 6 we found that BE was weaker for monocular viewing, suggesting that $\mathrm{BE}$ is stronger for pictorial stimuli seen binocularly. However, Experiment 7 found BE for both pictorial and nonpictorial stimuli. In Experiment 7, we report, for the first time, a test of BE using stereograms. Consistently in both Experiments 6 and 7, and contrary to expectations, BE did not increase with an increase of perceived depth in the scene. The fact that perceived depth was stronger for monocular viewing is an assumption based on the literature (Ames, 1925; Schlosberg, 1941), but for stereograms there is no doubt that observers noticed the depth information.

The fact that reducing the information available (monocular condition) leads to better performance may seem contradictory, but there are indeed other examples within picture perception. For instance, Yang and Kubovy (1999) found that binocular viewing increased variability and decreased sensitivity to perspective information. They explained this as a form of compensation. However, in the case of BE the issue is not one of level of performance but of a systematic bias.

As we discussed in the introduction, Intraub's (2004) preferred interpretation is in terms of an expected but occluded surround. However, Intraub (2002) herself, and more directly Hubbard (1995), have discussed how BE can be seen as an anticipatory aspect of spatial representation. If so, BE should not be affected by binocular or monocular viewing of pictures, or if anything it should increase with monocular viewing given that monocular viewing facilitates the perception of space in photographs. It seems therefore that BE may be an anticipatory aspect of the representation of a spatial configuration, that is, a scene, but unrelated to perception of space as such.

With respect to our attempt to clarify the meaning of close-up, we conclude that BE does not depend on the presence of magnification but is affected by changes in the target object when the information at the boundaries is kept constant. In other words, a close-up needs to be defined as a percept of the objects being near the observer, and such percept is induced by a combination of geometrical properties of the image. It is possible that the reason why multiple factors affect $\mathrm{BE}$ is because $\mathrm{BE}$ is a combination of more than one phenomenon. Two distinct varieties of BE may be at work: a BE germane to picture perception and a BE that applies to the general perception of spatial extent. Future research must show if this dichotomy is substantiated.

\section{References}

Ames, A., Jr. (1925). The illusion of depth from single pictures. Journal of the Optical Society of America, 10, 137-148.

Bertamini, M., Latto, R., \& Spooner, A. (2003). The Venus effect: Depiction of mirrors and mirror reflections in art. Perception, 32, 593-599.

Bertamini, M., \& Parks, T. E. (2005). On what people know about images on mirrors. Cognition, 98, 85-104.

Bertamini, M., Spooner, A., \& Hecht, H. (2003). Naive optics: Predicting and perceiving reflections in mirrors. Journal of Experimental Psychology: Human Perception and Performance, 29, 982-1002.

Bozzi, P. (1990). Fisica ingenua. Milan, Italy: Garzanti.

Candel, I., Merckelbach, H., \& Zandbergen, M. (2003). Boundary distor- tions for neutral and emotional pictures. Psychonomic Bulletin \& Review, 10, 691-695.

Croucher, C. J., Bertamini, M., \& Hecht, H. (2002). Naive optics: Understanding the geometry of mirror reflections. Journal of Experimental Psychology: Human Perception and Performance, 28, 546-562.

Cutting, J. E. (2003). Reconceiving perceptual space. In H. Hecht, M. Atherton, \& R. Schwartz (Eds.), Looking into pictures: An interdisciplinary approach to pictorial space (pp. 215-238). Boston: MIT Press.

Friedman, A. (1979). Framing pictures: The role of knowledge in automatized encoding and memory for gist. Journal of Experimental Psychology: General, 108, 316-355.

Gottesman, C. V., \& Intraub, H. (2002). Surface construal and the mental representation of scenes. Journal of Experimental Psychology: Human Perception and Performance, 28, 589-599.

Gottesman, C. V., \& Intraub, H. (2003). Constraints on spatial extrapolation in the mental representation of scenes: View-boundaries versus object-boundaries. Visual Cognition, 10, 875-893.

Hagen, M. (1980). The psychology of pictorial representation. New York: Academic Press.

Hagen, M., \& Giorgi, R. (1993). Where is the camera? Ecological Psychology, 5, 65-84.

Hecht, H., Bertamini, M., \& Gamer, M. (2005). Naive optics: Acting upon mirror reflections. Journal of Experimental Psychology: Human Perception and Performance, 31, 1023-1038.

Hecht, H., van Doorn, A. J., \& Koenderink, J. (1999). Compression of visual space in natural scenes and in their photographic counterparts. Perception \& Psychophysics, 61, 1269-1286.

Hochberg, J. (1986). Representation of motion and space in video and cinematic displays. In K. J. Boff, L. Kaufman, \& J. P. Thomas (Eds.), Handbook of perception and human performance (pp. 22.21-22.64). New York: Wiley.

Hubbard, T. L. (1995). Environmental invariants in the representation of motion: Implied dynamics and representational momentum, gravity, friction, and centripetal force. Psychonomic Bulletin \& Review, 2, 322338.

Intraub, H. (2002). Anticipatory spatial representations of natural scenes: Momentum without movement? Visual Cognition, 9, 93-119.

Intraub, H. (2004). Anticipatory spatial representation of 3D regions explored by sighted observers and a deaf-and-blind-observer. Cognition, 94, 19-37.

Intraub, H., Bender, R. S., \& Mangels, J. A. (1992). Looking at pictures but remembering scenes. Journal of Experimental Psychology: Learning, Memory, and Cognition, 18, 180-191.

Intraub, H., Gottesman, C. V., \& Bills, A. J. (1998). Effects of perceiving and imagining scenes on memory for pictures. Journal of Experimental Psychology: Learning, Memory, and Cognition, 24, 186-201.

Intraub, H., Gottesman, C. V., Willey, E. V., \& Zuk, I. J. (1996). Boundary extension for briefly glimpsed photographs: Do common perceptual processes result in unexpected memory distortions? Journal of Memory and Language, 35, 118-134.

Intraub, H., \& Richardson, M. (1989). Wide-angle memories of close-up scenes. Journal of Experimental Psychology: Learning, Memory, and Cognition, 15, 179-187.

Kaiser, M. K., Proffitt, D. R., Whelan, S. M., \& Hecht, H. (1992). The influence of animation on dynamical judgments. Journal of Experimental Psychology: Human Perception and Performance, 18, 669-690.

Kanizsa, G. (1979). Organization in vision. New York: Praeger.

Kennedy, J. M. (1974). A psychology of picture perception. San Francisco: Jossey-Bass.

Kubovy, M. (1986). The psychology of perspective and Renaissance art. Cambridge, England: Cambridge University Press.

Legault, E., \& Standing, L. (1992). Memory for size of drawings and photographs. Perceptual and Motor Skills, 75, 121.

Lumsden, E. A. (1980). Problems of magnification and minification: An 
explanation of the distortions of distance, slant, shape and velocity. In M. Hagen (Ed.), The psychology of pictorial representation (pp. 91135). New York: Academic Press.

Lumsden, E. A. (1983). Perception of radial distance as a function of magnification and truncation of depicted spatial layout. Perception \& Psychophysics, 33, 177-182.

McCloskey, M., Washburn, A., \& Felch, L. (1983). Naive physics: The straight-down belief and its origin. Journal of Experimental Psychology: Learning, Memory, and Cognition, 9, 636-649.

Prenz, P., \& Gerbino, W. (2004). Boundary extension: Proportional versus non-proportional transformations in visual memory. Perception, 33, 139.

Proffitt, D. R. (1999). Naïve physics. In R. A. Wilson \& F. C. Keil (Eds.), The MIT encyclopedia of the cognitive sciences (pp. 577-579). Cambridge, MA: MIT Press.

Rogers, S. (2003). Truth and meaning in pictorial space. In H. Hecht, R. Schwartz \& M. Atherton (Eds.), Looking into pictures: An interdisciplinary approach to pictorial space (pp. 119-163). Cambridge, MA: MIT Press.
Schlosberg, H. (1941). Stereoscopic depth from single pictures. American Journal of Psychology, 54, 601-605.

Seamon, J. G., Schlegel, S. E., Hiester, P. M., Landau, S. M., \& Blumenthal, B. F. (2002). Misremembering pictured objects: People of all ages demonstrate the boundary extension illusion. American Journal of Psychology, 115, 151-167.

Simons, D. J. (2000). Current approaches to change blindness. Visual Cognition, 7, 1-15.

TNO. (1972). Test for stereoscopic vision (10th ed.). Utrecht, the Netherlands: Lameris Tech.

Yang, T. L., \& Kubovy, M. (1999). Weakening the robustness of perspective: Evidence for a modified theory of compensation in picture perception. Perception \& Psychophysics, 61, 456-467.

Received May 13, 2004 Revision received April 25, 2005

Accepted April 26, 2005

\section{Call for Nominations}

The Publications and Communications (P\&C) Board has opened nominations for the editorships of Behavioral Neuroscience, JEP: Applied, JEP: General, Neuropsychology, Psychological Methods, and Psychology and Aging for the years 2008-2013. John F. Disterhoft, PhD; Phillip L. Ackerman, PhD; D. Stephen Lindsay, PhD; James T. Becker, PhD; Stephen G. West, PhD; and Rose T. Zacks, PhD, respectively, are the incumbent editors.

Candidates should be members of APA and should be available to start receiving manuscripts in early 2007 to prepare for issues published in 2008. Please note that the P\&C Board encourages participation by members of underrepresented groups in the publication process and would particularly welcome such nominees. Self-nominations also are encouraged.

Search chairs have been appointed as follows:

- Behavioral Neuroscience: Linda P. Spear, PhD, and J. Gilbert Benedict, PhD

- JEP: Applied: William C. Howell, PhD

- JEP: General: Peter A. Ornstein, PhD

- Neuropsychology: Susan H. McDaniel, PhD, and Robert G. Frank, PhD

- Psychological Methods: Mark Appelbaum, PhD

- Psychology and Aging: David C. Funder, PhD, and Leah L. Light, PhD

Candidates should be nominated by accessing APA's EditorQuest site on the Web. Using your Web browser, go to http://editorquest.apa.org. On the Home menu on the left, find Guests. Next, click on the link "Submit a Nomination," enter your nominee's information, and click "Submit."

Prepared statements of one page or less in support of a nominee can also be submitted by e-mail to Karen Sellman, P\&C Board Search Liaison, at ksellman@apa.org.

Deadline for accepting nominations is January 20, 2006, when reviews will begin. 